\title{
A CENTRAL LIMIT THEOREM FOR UNIFORMLY BOUNDED ORTHONORMAL SYSTEMS $\left.{ }^{1}\right)$
}

\author{
BY \\ GEORGE W. MORGENTHALER
}

1. Introduction. Let $\left\{\xi_{n}(x)\right\}$ be a sequence of random variables on a probability space $(\bar{X}, \bar{S}, m)$, i.e., $\bar{S}$ is a $\sigma$-algebra of subsets of the basic space $\bar{X}$, and $m$ is a measure on $\bar{S}, m(\bar{X})=1$. Form the new random variables

$$
\frac{S_{N}(x)-\mu\left(S_{N}\right)}{\sigma\left(S_{N}\right)}, \quad \quad N=1,2,3, \cdots,
$$

where $S_{N}(x)=\sum_{i=1}^{N} \xi_{i}(x)$ and where for any random variable $\xi(x)$ we define $\mu(\xi)=\int_{-\infty}^{\infty} t d F(t)$ and $\sigma(\xi)=\left[\int_{-\infty}^{\infty}(t-\mu(\xi))^{2} d F(t)\right]^{1 / 2}$, the mean and standard deviation respectively of $\xi(x)$ with respect to its distribution function $F(t)$. The Central Limit Problem of mathematical statistics may then be stated as:

(1.2) Determine necessary and sufficient conditions under which the distribution functions $\left\{F_{N}(t)\right\}$ associated with the random variables (1.1) will converge pointwise to the normal or Gaussian distribution $G(t)=(2 \pi)^{-1 / 2} \int_{-\infty}^{t} e^{-u^{2} / 2} d u$.

A history of the progress in solving this problem is found in a recent article by Loève [6]( $\left.{ }^{2}\right)$. The work has separated naturally into two parts: the case of independent random variables solved completely by Lévy, Lindeberg, and Feller; and the dependent case for which one of the best known results is due to $\mathrm{S}$. Bernstein. We shall be concerned here with partial solutions to the Central Limit Problem in the special case of certain sequences of real-valued measurable functions on $(0,1)$. Lebesgue measure of a set $E$ shall be denoted throughout by $m(E)$.

One version of the Central Limit Theorem (Lindeberg [5]) for a sequence $\left\{f_{k}(x)\right\}$ of independent functions states that

if $\int_{0}^{1} f_{k}(x) d x=0, \int_{0}^{1} f_{k}^{2}(x) d x=1, k=1,2,3, \cdots$, and if

$\epsilon>0 \cdot \supset \cdot \exists M=M(\epsilon) \ni \int_{\left\{x \in[0,1] \mid \gamma_{k}^{2}(x)>M\right\}} f_{k}^{2}(x) d x<\epsilon, \quad k=1,2,3, \cdots$,

then $\sum_{k=1}^{N} f_{k}(x) / N^{1 / 2}$ is distributed asymptotically normally with mean value zero and dispersion 1.

Presented to the Society, April 26, 1952; received by the editors March 2, 1954.

(1) The work on which this paper is based was supported by the Office of Naval Research. The paper is essentially the first part of a dissertation submitted in June 1953 in partial fulfillment of the requirements for the Ph.D. degree at the University of Chicago. The author wishes to express his gratitude to Professor Antoni Zygmund for his helpful suggestions and criticism throughout this work.

(2) The numbers in brackets refer to the bibliography which appears at the end. 
The investigation in the case when the functions are not necessarily independent has proceeded along two lines. Kac [4] established the following:

(1.3) Theorem. Let $f(x)$ be a periodic function of class Lipschitz- $\alpha$ with $\alpha \geqq 1 / 2$ and $\int_{0}^{1} f(x) d x=0$. If $\left\{c_{k}\right\}$ is an arbitrary sequence of real numbers for which

(a) $\left(\sum_{k=1}^{N} c_{k}^{2}\right)^{1 / 2}=C_{N} \rightarrow \infty$ as $N \rightarrow \infty$,

(b) $c_{N}=O(1)$,

(c) $\lim _{N \rightarrow \infty} \int_{0}^{1}\left(S_{N}(x) / C_{N}\right)^{2} d x=\sigma^{2}>0$, where $S_{N}(x)=\sum_{k=1}^{N} c_{k} f\left(2^{b_{k}} x\right)$ and $\left\{b_{k}\right\}$ is any sequence of integers with $\left(b_{k+1}-b_{k}\right)$ increasing, then $S_{N}(x) / C_{N}$ is distributed asymptotically normally with mean value 0 and dispersion $\left(\int_{0}^{1} f^{2}(x) d x\right)^{1 / 2}$.

The other direction of approach has been to specify the functional form of the $f_{k}(x)$. A number of authors have considered the case of a lacunary sequence of trigonometric functions. The most general result here is due to Salem and Zygmund [9] who state the following theorem.

(1.4) Theorem. Let $S_{N}(x)$ denote the Nth partial sum of the lacunary trigonometric series $\sum_{k=1}^{N}\left(a_{k} \cos n_{k} x+b_{k} \sin n_{k} x\right), n_{k}$ integral, $n_{k+1} / n_{k}>q>1$, and let $a_{1}, a_{2}, a_{3}, \cdots ; b_{1}, b_{2}, b_{3}, \cdots$ be arbitrary sequences of real numbers for which, as $N \rightarrow \infty$,

(a) $C_{N}=\left[1 / 2\left(a_{1}^{2}+b_{1}^{2}+a_{2}^{2}+b_{2}^{2}+\cdots+a_{N}^{2}+b_{N}^{2}\right)\right]^{1 / 2} \rightarrow \infty$,

(b) $\left(a_{N}^{2}+b_{N}^{2}\right)^{1 / 2} / C_{N} \rightarrow 0$.

Then, for any set $E \subset[0,2 \pi]$ of positive measure, the distribution functions

$$
F_{N}(y: E)=\frac{m\left(\left\{x \in E \mid S_{N}(x) / C_{N} \leqq y\right\}\right)}{m(E)}, \quad N=1,2,3, \cdots,
$$

tend to the Gaussian distribution with mean 0 and dispersion 1.

This result has been extended by the authors to the case of the infinite interval (using relative measure) and the case of nonintegral $n_{k}$.

The main problem of this paper is to establish an analogue of the SalemZygmund theorem for general uniformly bounded orthonormal systems. If $\left\{\theta_{n}(x)\right\}$ is a sequence of real-valued functions in $L^{2}(a, b)$ for which

$$
\int_{a}^{b} \theta_{m}(x) \theta_{n}(x) d x= \begin{cases}0, & m \neq n, \\ 1, & m=n,\end{cases}
$$

the system is termed a real orthonormal system.

In $\$ 2$ below a theorem of Banach and Saks on average strong convergence in $L^{p}$ is generalized. This basic lemma is then used in $\$ 3$ to establish the following result for real-valued, uniformly bounded, orthonormal systems.

(1.6) THEOREM II. Let $\left\{\phi_{n}(x)\right\}$ be a uniformly bounded orthonormal system of real-valued functions on a finite interval $[a, b]$. Then there exists a subsequence 
$\left\{\phi_{n_{k}}(x)\right\}$ and a real-valued function $f(x)$ on $[a, b], \int_{a}^{b} f(x) d x=1,0 \leqq f(x) \leqq M^{2}$, $M$ the uniform bound of $\left\{\phi_{n}(x)\right\}$, such that for any measurable set $E \subset[a, b]$ with $m(E)>0$ and any $\left\{a_{k}\right\}$ an arbitrary sequence of real numbers satisfying:

(a) $A_{N}=\left(a_{1}^{2}+a_{2}^{2}+\cdots+a_{N}^{2}\right)^{1 / 2} \rightarrow \infty$ as $N \rightarrow \infty$,

(b) $a_{N}=o\left(A_{N}\right)$,

the distribution functions

$$
F_{N}(y: E)=\frac{m\left(\left\{x \in E \mid \sum_{k=1}^{N} a_{k} \phi_{n_{k}}(x) / A_{N} \leqq y\right\}\right)}{m(E)}, \quad N=1,2,3, \cdots,
$$

converge to a limiting distribution $F(y: E)$ at each point of continuity of the latter, and the characteristic function of $F(y: E)$ is

$$
\Phi(\lambda: E)=m(E)^{-1} \int_{E} e^{-\lambda^{2} f(x) / 2} d x .
$$

Counterexamples are also given in $\$ 3$ to show that a true Gaussian distribution cannot always be obtained as the limit $F(y: E)$ and so in a sense this theorem cannot be strengthened. The appearance of (1.7) suggests the title Pseudo-Gaussian Distribution Theorem for Theorem II. Theorem III asserts that for every nonnegative, bounded function $f(x)$ on $[a, b]$ with $\int_{a}^{b} f(x) d x=1$ there exists a uniformly bounded orthonormal system for which, when Theorem II is applied to this system, $f(x)$ appears in the exponent of $\Phi(\lambda: E)$ in (1.7). The section ends with a generalized result of the type obtained by Kac (see (1.3)) for the sequence $\{f(n x)\}$ where $f(x)$ is any bounded, periodic function of mean value zero on a finite interval $[a, b]$. The hypotheses of the result are less restrictive than those of $\mathrm{Kac}$ in that only boundedness rather than membership in a Lipschitz class is assumed for $f(x)$. The conclusion is somewhat less precise in that the existence of a subsequence $\left\{f\left(n_{k} x\right)\right\}$ is asserted for which the Central Limit Theorem holds rather than the assertion of its validity for the particular sequence $\left\{f\left(2^{b_{k}} x\right)\right\}$ of Kac's result.

The final section contains Theorem V, the analogue of Theorem II for complex-valued orthonormal systems. The resulting distributions are twodimensional, $\left\{F_{N}(y, z: E)\right\}$, and the limit distribution has characteristic function

$$
\Phi(\lambda, \mu: E)=m(E)^{-1} \int_{E} \exp \left(-1 / 2\left(\lambda^{2} R(x)+2 \lambda \mu Q(x)+\mu^{2} S(x)\right)\right) d x
$$

where $R(x), Q(x), S(x)$ are real-valued, $0 \leqq R(x),|Q(x)|, S(x) \leqq M^{2}$, and $\int_{a}^{b}(R(x)+S(x)) d x=1$. Moreover, given nonnegative, bounded functions $R(x)$ and $S(x)$ satisfying $\int_{a}^{b}(R(x)+S(x)) d x=1$ there exists a complex orthonormal system on $[a, b]$ for which this $R(x)$ and $S(x)$ appear in the exponent in the corresponding $\Phi(\lambda, \mu: E)$ of (1.8) and the function $Q(x)$ is zero a.e. 
It is clear that Theorem II is a special case of Theorem V. It may be thought, therefore, that economy and elegance would demand that the complex result be stated and proved without mention of the special case of real systems. The examples and remarks included after each result, however, are of some interest and the main ideas of the proof are more efficiently presented in the case of real systems. Thus, in order to spare the reader considerable complication of notation and to save printed space, the details of the proof for real systems shall be spelled out and the discussion in the complex case (the proof embodies the same ideas as for real systems) shall center around the examples only.

2. On a result of Banach and Saks. Banach and Saks [1] observed that weak convergence of a sequence of functions of bounded norm in $L^{p}$ implies average strong convergence of a subsequence of the functions. Specifically,

Theorem. If $\left\{\psi_{n}(x)\right\} \in L^{p}(0,1), p>1, \psi_{n}(x)$ bounded in norm, there exists a subsequence $\left\{\psi_{n_{k}}(x)\right\}$ and a function $f(x) \in L^{p}(0,1)$ such that

$$
\lim _{N \rightarrow \infty} \int_{0}^{1}\left|\sum_{k=1}^{N} \psi_{n_{k}}(x) / N-f(x)\right|^{p} d x=0 .
$$

Although the generalization of this result plays the role of a lemma to support the main result of this paper, it has intrinsic interest.

Theorem I. If $\left\{\psi_{n}(x)\right\} \in L^{p}(a, b), p>1, \psi_{n}(x)$ bounded in norm, there exists a subsequence $\left\{\psi_{n_{k}}(x)\right\}$ and a function $f(x)$ in $L^{p}(a, b)$ such that for any sequence $\left\{\lambda_{i}\right\}$ of nonnegative real numbers satisfying:

(a) $A_{N}=\left(\lambda_{1}+\lambda_{2}+\cdots+\lambda_{N}\right) \rightarrow \infty$ as $N \rightarrow \infty$,

(b) $\lambda_{N}=o\left(A_{N}\right)$

$$
\lim _{N \rightarrow \infty} \int_{a}^{b}\left|\sum_{k=1}^{N} \lambda_{k} \psi_{n_{k}}(x) / A_{N}-f(x)\right|^{p} d x=0
$$

Proof. The first lemma is well known.

Lemma 1. Conditions (a) and (b) imply $\max _{1 \leqq k \leqq N}\left(\lambda_{k}\right) / A_{N} \rightarrow 0$ as $N \rightarrow \infty$.

Lemma 2 (F. RIEsz [8]). If the functions $\left\{\psi_{n}(x)\right\} \in L^{p}(a, b), p>1$, are bounded in norm, there exists a subsequence $\left\{\psi_{n_{k}}(x)\right\}$ and a function $f(x)$ $\in L^{p}(a, b)$ which is the weak limit of $\left\{\psi_{n_{k}}(x)\right\}$, i.e., for every $h(x) \in L^{q}(a, b)$, $1 / p+1 / q=1, \lim _{k \rightarrow \infty} \int_{a}^{b} h(x) \psi_{n_{k}}(x) d x=\int_{a}^{b} h(x) f(x) d x$.

The crucial number-theoretic lemma of the Banach-Saks paper may be stated as follows:

Lemma 3. For $p>1$ and any real numbers $a, b$

$$
|a+b|^{p} \leqq|a|^{p}+p|a|^{p-1}(\operatorname{Sgn} a) b+\sum_{i=2}^{[p]} C_{p, i}|a|^{p-i}|b|^{i}+A|b|^{p}
$$


where $A$ designates a positive constant independent of $a$ and $b$.

The proof of the theorem may be carried out immediately for general $p$ if it is only observed that for $p<2$ the third term on the right side of (2.2) vanishes from the discussion. Also there is no loss of generality in taking $[a, b]$ as $[0,1]$ throughout.

Given the initial sequence $\left\{\psi_{n}(x)\right\}$ of the theorem, we apply Lemma 2 and obtain a subsequence $\left\{\psi_{n_{k}}(x)\right\}$ and a function $f(x) \in L^{p}(0,1)$ such that $f(x)$ is the weak limit of $\left\{\psi_{n_{k}}\right\}$. It is no loss of generality to assume $f(x) \equiv 0$. If, to simplify notation, we denote this subsequence which converges weakly to $f(x) \equiv 0$ by $\left\{\psi_{n}(x)\right\}$, it is necessary to devise a scheme for choosing a further subsequence $\left\{\psi_{n_{k}}(x)\right\}$ having the property of the theorem. Assuming $\psi_{n_{1}}=\psi_{1}$, $\psi_{n_{2}}, \psi_{n_{3}}, \cdots, \psi_{n_{j}}$ have been suitably choosen, we shall specify how $\psi_{n_{j+1}}$ is to be selected.

Set $S_{j}(x)=\sum_{i=1}^{j} \lambda_{i} \psi_{n_{i}}(x)$ and apply Lemma 3 with $a=S_{j}(x), b=\lambda_{j+1}$ $\cdot \psi_{n_{j+1}}(x)$. An integration yields

$$
\begin{aligned}
\int_{0}^{1} \mid & \left.S_{j+1}(x)\right|^{p} d t \\
& \leqq \\
& \int_{0}^{1}\left|S_{j}(t)\right|^{p} d t+p \lambda_{j+1} \int_{0}^{1}\left|S_{j}(t)\right|^{p-1}\left(\operatorname{Sgn} S_{j}(t)\right) \psi_{n_{j+1}}(t) d t \\
& \quad+\sum_{i=2}^{[p]} C_{p, i} \lambda_{j+1}^{i} \int_{0}^{1}\left|S_{j}(t)\right|^{p-i}\left|\psi_{n_{j}}(t)\right|^{i} d t+\lambda_{j+1}^{p} A \int_{0}^{1}\left|\psi_{n_{j+1}}(t)\right|^{p} d t .
\end{aligned}
$$

For $p \geqq 2$ the third term on the right may be majorized as follows. By assumption $\left[\int_{0}^{1}\left|\psi_{n}(t)\right|^{p} d t\right]^{1 / p} \leqq K$, all $n$, and hence by Hölder's inequality

$$
\begin{aligned}
\sum_{i=2}^{[p]} C_{p, i} \lambda_{j+i}^{i} \int_{0}^{1}\left|S_{j}(t)\right|^{p-i} \mid & \left.\psi_{n_{j+1}}(t)\right|^{i} d t \\
& \leqq \sum_{i=2}^{[p]} C_{p, i} \lambda_{j+1}^{i} K^{i}\left[\int_{0}^{1}\left|S_{j}(t)\right|^{p} d t\right]^{(p-i) / p} .
\end{aligned}
$$

But $\left[\int_{0}^{1}\left|S_{j}(t)\right|^{p} d t\right]^{1 / p} \leqq \sum_{k=1}^{1}\left[\int_{0}^{j} \lambda_{k}^{p}\left|\psi_{n_{k}}(t)\right|^{p} d t\right]^{1 / p} \leqq \sum_{k=1}^{j} \lambda_{k} K=K A_{j}$ by Minkowski's inequality and upon substitution the right side of (2.4) is majorized by

$$
\leqq \sum_{i=2}^{[p]} C_{p, i} \lambda_{j+1}^{i} K^{i}\left[K^{p-i} A_{j}^{p-i}\right]=K^{p} \sum_{i=2}^{[p]} C_{p, i} \lambda_{j+1}^{i} A_{j}^{p-i} .
$$

Let $b=i-2$ and then this last expression is majorized by

$$
\leqq K^{p} \lambda_{j+1}^{2} A_{j}^{p-[p]}\left[\sum_{b=0}^{[p]-2} C_{[p]-2, b} \lambda_{j+1}^{b} A_{j}^{[p]-2-b}\right]\left[\max _{0 \leqq b \leqq p]-2} \frac{C_{p, b+2}}{C_{[p]-2, b}}\right] .
$$


Since $p$ is fixed, [ $\left.\max _{0 \leqq b \leqq[p]-2} C_{p, b+2} / C_{[p]-2, b}\right]$ is a constant, say $R$, and so the right side of (2.4) is majorized by

$$
\begin{aligned}
& \leqq R K^{p} \lambda_{j+1}^{2} A_{j}^{p-[p]}\left[\lambda_{j+1}+A_{j}\right]^{[p]-2} \\
& \leqq R K^{p} \lambda_{j+1}^{2} A_{j+1}^{p-2}
\end{aligned}
$$

Using this estimate in (2.3) and employing the identity $|a|^{p-1} \operatorname{Sgn} a$ $=a|a|^{p-2}$ we have

$$
\begin{aligned}
\int_{0}^{1}\left|S_{j+1}(t)\right|^{p} d t \leqq & \int_{0}^{1}\left|S_{j}(t)\right|^{p} d t+p \lambda_{j+1} \int_{0}^{1}\left|S_{j}(t)\right|^{p-2} S_{j}(t) \psi_{n_{j+1}}(t) d t \\
& +R K^{p} \lambda_{j+1}^{2} A_{j+1}^{p-2}+A K^{p} \lambda_{j+1}^{p} .
\end{aligned}
$$

The function $\psi_{n_{j+1}}(x)$ will be selected so as to make the second term on the right small compared to $A_{j}^{p-1}$, independently of sequence $\left\{\lambda_{k}\right\}$.

Consider now the sequence of functions in $j$-space defined by

$$
f_{m}\left(\alpha_{1}, \alpha_{2}, \cdots, \alpha_{j}\right)=\int_{0}^{1}\left|\sum_{i=1}^{j} \alpha_{i} \psi_{n_{i}}(t)\right|^{p-2}\left[\sum_{i=1}^{j} \alpha_{i} \psi_{n_{i}}(t)\right] \psi_{m}(t) d t, \quad m>n_{j}
$$

where $\psi_{n_{1}}, \psi_{n_{2}}, \cdots, \psi_{n_{j}}$ are the fixed functions previously chosen. Now the coefficient of $\psi_{m}(t)$ in the integrand is observed to have absolute value $\left|\sum_{i=1}^{j} \alpha_{i} \psi_{n_{i}}(t)\right|^{p-1}$ and hence, for any numbers $\alpha_{1}, \alpha_{2}, \cdots, \alpha_{j}$ this coefficient belongs to $L^{p / p-1}$. Since sequence $\left\{\psi_{m}(t)\right\}$ converges weakly to zero, it follows that for each point $\left(\alpha_{1}, \alpha_{2}, \cdots, \alpha_{j}\right)$ on the face of the $j$-hedron $\sum_{i=1}^{j} \alpha_{i}=1$ lying in the first "quadrant" of $j$-space, i.e., $\alpha_{i} \geqq 0, i=1,2,3, \cdots, j$,

$$
\lim _{m \rightarrow \infty} f_{m}\left(\alpha_{1}, \alpha_{2}, \cdots, \alpha_{j}\right)=0 .
$$

It may further be verified that the $\left\{f_{m}\left(\alpha_{1}, \alpha_{2}, \cdots, \alpha_{j}\right)\right\}$ form an equicontinuous set of functions at each of these points $\left(\alpha_{1}, \alpha_{2}, \cdots, \alpha_{j}\right)$. It follows that the functions $\left\{f_{m}\left(\alpha_{1}, \alpha_{2}, \cdots, \alpha_{j}\right)\right\}$ converge uniformly to zero on the closed bounded subset of $j$-space represented by the face of the $j$-hedron in the first quadrant. This affords the selection scheme required. Choose the integer $n_{j+1}$ so large that

$$
\left.\left|\int_{0}^{1}\right| \sum_{i=1}^{j} \alpha_{i} \psi_{n_{i}}(t)\right|^{p-2}\left[\sum_{i=1}^{j} \alpha_{i} \psi_{n_{i}}(t)\right] \psi_{n_{j+1}}(t) d t \mid \leqq 1 / 2^{j+1}
$$

for all points $\left(\alpha_{1}, \alpha_{2}, \cdots, \alpha_{j}\right)$ satisfying $\sum_{i=1}^{j} \alpha_{i}=1, \alpha_{i} \geqq 0,1 \leqq i \leqq j$.

Suppose the subsequence $\left\{\psi_{n_{k}}(x)\right\}$ is so selected. For any sequence $\left\{\lambda_{k}\right\}$, $\lambda_{k} \geqq 0$, assume $j$ so large that $A_{j} \neq 0$. We may then divide (2.5) by $A_{j+1}^{p}$. It is observed, however, that the right side of the resulting inequality will only be increased if the integral of the second term is replaced by its absolute value 
and if in its denominator the factor $A_{j+1}^{p-1}$ is replaced by the lesser quantity $A_{j}^{p-1}$. The resulting expression is

$$
\begin{aligned}
\int_{0}^{1}\left|\frac{S_{j+1}(t)}{A_{j+1}}\right|^{p} d t \leqq & \int_{0}^{1}\left|\frac{S_{j}(t)}{A_{j+1}}\right|^{p} d t \\
& +\left.\frac{p \lambda_{j+1}}{A_{j+1}}\left|\int_{0}^{1}\right| \frac{S_{j}(t)}{A_{j}}\right|^{p-2}\left[\frac{S_{j}(t)}{A_{j}}\right] \psi_{n_{j+1}}(t) d t \mid \\
& +\frac{R K^{p} \lambda_{j+1}^{2}}{A_{j+1}^{2}}+\frac{A K^{p} \lambda_{j+1}^{p}}{A_{j+1}^{p}} .
\end{aligned}
$$

In the first and second factors of the integrand in the second term on the right-hand side of (2.8) the coefficients of $\psi_{n_{1}}(t), \psi_{n_{2}}(t), \cdots, \psi_{n_{j}}(t)$ are $\lambda_{1} / A_{j}, \lambda_{2} / A_{j}, \cdots, \lambda_{j} / A_{j}$ respectively. But $\left(\lambda_{1} / A_{j}, \lambda_{2} / A_{j}, \cdots, \lambda_{j} / A_{j}\right)$ is a point on the face of the $j$-hedron in $j$-space and by (2.7) the second term does not exceed $p \lambda_{j+1} / A_{j+1} 2^{j+1}$.

Make this replacement in the second term on the right and use inequality (2.8) as a recursion formula, applying it in all $(j-1)$ times. (Of course, if say $A_{j \rightarrow \nu}=0$, then $\lambda_{1}=\lambda_{2}=\cdots=\lambda_{j \rightarrow \nu}=0$ and $S_{i}(t)=0, i \leqq j-\nu$, so that we would apply the formula only $(j-\nu+1)$ times.) If e.g., $\lambda_{1} \neq 0$, we have

$$
\begin{aligned}
& \int_{0}^{1}\left|\frac{S_{j+1}(t)}{A_{j+1}}\right|^{p} d t \leqq A K^{p}\left[\left(\frac{\lambda_{j+1}}{A_{j+1}}\right)^{p}\right]+\frac{p \lambda_{j+1}}{A_{j+1}}\left(\frac{1}{2^{j+1}}\right)+\frac{R K^{p} \lambda_{j+1}^{2}}{A_{j+1}^{2}} \\
&+\left(\frac{A_{j}}{A_{j+1}}\right)^{p}\left[\frac{A K^{p} \lambda_{j}^{p}}{A_{j}^{p}}+\frac{p \lambda_{j}}{A_{j}}\left(\frac{1}{2^{j}}\right)+\frac{R K^{p} \lambda_{j}^{2}}{A_{j}^{2}}\right. \\
&\left.+\int_{0}^{1}\left|\frac{S_{j-1}(t)}{A_{j}}\right|^{p} d t\right] .
\end{aligned}
$$

But

$$
\left(\frac{A_{j}}{A_{j+1}}\right)^{p} \frac{p \lambda_{j}}{A_{j}}\left(\frac{1}{2^{i}}\right) \leqq \frac{p \lambda_{j}}{A_{j+1}}\left(\frac{1}{2^{i}}\right)
$$

since $\left(A_{j} / A_{j+1}\right)^{p-1} \leqq 1$. Also when $p \geqq 2$

$$
\left(\frac{A_{j}}{A_{j+1}}\right)^{p} \frac{R K^{p} \lambda_{j}^{2}}{A_{j}^{2}} \leqq\left(\frac{A_{j}}{A_{j+1}}\right)^{p-2} \frac{R K^{p} \lambda_{j}^{2}}{A_{j+1}^{2}} \leqq \frac{R K^{p} \lambda_{j}^{2}}{A_{j+1}^{2}} .
$$

In the case $p<2$ it has already been mentioned that the second term on the right is missing in the basic inequality (2.2), (2.3), and subsequent expressions.

With these remarks, the right side of the last inequality is 


$$
\begin{aligned}
\leqq & A K^{p}\left[\left(\frac{\lambda_{j+1}}{A_{j+1}}\right)^{p}+\left(\frac{\lambda_{j}}{A_{j+1}}\right)^{p}\right]+\frac{p}{A_{j+1}}\left[\frac{\lambda_{j+1}}{2^{j+1}}+\frac{\lambda_{j}}{2^{j}}\right] \\
& +R K^{p}\left[\left(\frac{\lambda_{j+1}}{A_{j+1}}\right)^{2}+\left(\frac{\lambda_{j}}{A_{j+1}}\right)^{2}\right] \\
& +\left(\frac{A_{j-1}}{A_{j+1}}\right)^{p}\left[\frac{A K^{p} \lambda_{j-1}^{p}}{A_{j-1}^{p}}+\frac{p \lambda_{j-1}}{A_{j-1}}\left(\frac{1}{2^{j-1}}\right)+\frac{R K^{p} \lambda_{j-1}^{2}}{A_{j-1}^{2}}+\int_{0}^{1}\left|\frac{S_{j-2}(t)}{A_{j-1}}\right|^{p} d t\right] .
\end{aligned}
$$

Continuing to repeat the simplification just employed, and factoring out, we are led to

$$
\begin{aligned}
& \int_{0}^{1}\left|\frac{S_{j+1}(t)}{A_{j+1}}\right|^{p} d t \leqq A K^{p}\left[\frac{\max _{1 \leqq 1 \leqq j+1}\left(\lambda_{i}\right)}{A_{j+1}}\right]^{p-1}\left[\frac{\lambda_{j+1}}{A_{j+1}}+\frac{\lambda_{j}}{A_{j+1}}+\cdots+\frac{\lambda_{2}}{A_{j+1}}\right] \\
& +\left[p \frac{\max _{1 \leqq i \leqq j+1}\left(\lambda_{i}\right)}{A_{j+1}}\right]\left[\frac{1}{2^{j+1}}+\frac{1}{2^{j}}+\frac{1}{2^{j-1}}+\cdots+\frac{1}{2^{2}}\right] \\
& +R K^{p}\left[\frac{\max _{1 \leqq i \leqq j+1}\left(\lambda_{i}\right)}{A_{j+1}}\right]\left[\frac{\lambda_{j+1}}{A_{j+1}}+\frac{\lambda_{j}}{A_{j+1}}+\cdots+\frac{\lambda_{2}}{A_{j+1}}\right] \\
& +\left(\frac{A_{2}}{A_{j+1}}\right)^{p}\left(\frac{\int_{0}^{1}\left|\lambda_{1} \psi_{n_{1}}(t)\right|^{p} d t}{A_{2}^{p}}\right) \\
& \leqq\left[K^{p}\left(\frac{\max _{1 \leqq i \leqq j+1}\left(\lambda_{i}\right)}{A_{j+1}}\right)^{p-1}+p\left(\frac{\max _{1 \leqq i \leqq j+1}\left(\lambda_{i}\right)}{A_{j+1}}\right)\right. \\
& \left.+R K^{p}\left(\frac{\max _{1 \leqq i \leqq j+1}\left(\lambda_{i}\right)}{A_{j+1}}\right)+\frac{\lambda_{1}^{p} K^{p}}{A_{j+1}^{p}}\right] .
\end{aligned}
$$

By application of Lemma 1 and with $A_{j+1} \rightarrow \infty$, it is evident that this quantity is small with large $j$, and thus the theorem is proved.

Corollary. Any subsequence $\left\{\psi_{n_{k_{j}}}(x)\right\}$ of the subsequence $\left\{\psi_{n_{k}}(x)\right\}$ provided by Theorem I still enjoys the property of Theorem I with respect to the same function $f(x)$. That is, given any nonnegative sequence of reals $\left\{\lambda_{j}\right\}$ satisfying

(a) $A_{N}=\left(\lambda_{1}+\lambda_{2}+\cdots+\lambda_{N}\right) \rightarrow \infty$ as $N \rightarrow \infty$,

(b) $\lambda_{N}=o\left(A_{N}\right)$, then

$$
\int_{0}^{1}\left|\sum_{j=1}^{N} \frac{\lambda_{j} \psi_{n_{k_{j}}}(x)}{A_{N}}-f(x)\right|^{p} d x \rightarrow 0 \quad \text { as } N \rightarrow \infty
$$


Proof. Take any sequence $\left\{\lambda_{j}\right\}$ satisfying the conditions (a) and (b). Define a sequence $\left\{\bar{\lambda}_{k}\right\}$ by the relations

$$
\bar{\lambda}_{k}= \begin{cases}\lambda_{j} & \text { if } \psi_{n_{k}} \equiv \psi_{n_{k_{j}}} \\ 0 & \text { elsewhere }\end{cases}
$$

and set $\bar{A}_{M}=\bar{\lambda}_{1}+\bar{\lambda}_{2}+\cdots+\bar{\lambda}_{M}$. Then $\left\{\bar{\lambda}_{k}\right\}$ is a nonnegative sequence for which $\bar{A}_{M} \rightarrow \infty$ and $\bar{\lambda}_{M}=o\left(\bar{A}_{M}\right)$ because of the properties of $\left\{\lambda_{j}\right\}$. Hence $\left\{\bar{\lambda}_{k}\right\}$ is an admissible sequence in Theorem I. Then

$$
\int_{0}^{1}\left|\sum_{k=1}^{M} \frac{\bar{\lambda}_{k} \psi_{n_{k}}(x)}{\bar{A}_{M}}-f(x)\right|^{p} d x \rightarrow 0 \quad \text { as } M \rightarrow \infty
$$

with $f(x)$ being the same $f(x)$ as in Theorem I. Also for each integer $p=N$,

$$
\frac{\sum_{j=1}^{p} \lambda_{j} \psi_{n_{j}}(x)}{A_{p}} \equiv \frac{\sum_{k=1}^{k_{p}} \bar{\lambda}_{k} \psi_{n_{k}}}{\bar{A}_{k_{p}}}
$$

thereby proving the corollary.

3. A pseudo-Gaussian distribution theorem for real orthonormal sequences. Counter-examples will be given to show that the form of the limiting distribution in the following theorem need not be truly Gaussian.

THEOREM II. Let $\left\{\phi_{n}(x)\right\}$ be a uniformly bounded orthonormal system of real-valued functions on a finite interval $[a, b]$. Then there exists a subsequence $\left\{\phi_{n_{k}}(x)\right\}$ and a real-valued function $f(x)$ on $[a, b], \int_{a}^{b} f(x) d x=1,0 \leqq f(x) \leqq M^{2}$, $M$ the uniform bound of $\left\{\phi_{n}(x)\right\}$, such that for any measurable set $E \subset[a, b]$ with $m(E)>0$ and any $\left\{a_{k}\right\}$ an arbitrary sequence of real numbers satisfying:

(a) $A_{N}=\left(a_{1}^{2}+a_{2}^{2}+\cdots+a_{N}^{2}\right)^{1 / 2} \rightarrow \infty$ as $N \rightarrow \infty$,

(b) $a_{N}=o\left(A_{N}\right)$,

the distribution functions

$$
\begin{aligned}
\left.F_{N}(y: E)=m\left(\left\{x \in E \mid \sum_{k=1}^{N} a_{k} \phi_{n_{k}}(x) / A_{N} \leqq y\right\}\right)\right) / m(E), \\
\quad N=1,2,3, \cdots,
\end{aligned}
$$

converge to a limiting distribution $F(y: E)$ at each point of continuity of the latter, and the characteristic function of $F(y: E)$ is

$$
\Phi(\lambda: E)=m(E)^{-1} \int_{E} e^{-\lambda^{2} f(x) / 2} d x .
$$

Proof. By the continuity theorem for characteristic functions of distributions it is sufficient to show the existence of a subsequence $\left\{\phi_{n_{k}}(x)\right\}$ and function $f(x), \int_{0}^{1} f(x) d x=1,0 \leqq f(x) \leqq M^{2}$, such that for any sequence $\left\{a_{k}\right\}$ admissible under the conditions of the theorem and for any set $E \subset[a, b], m(E)>0$, 
the characteristic functions of the distributions $\left\{F_{N}(y: E)\right\}$,

$$
\begin{array}{r}
\Phi_{N}(\lambda: E)=\int_{-\infty}^{\infty} e^{i \lambda} d F_{N}(y: E)=\frac{1}{m(E)} \int_{E} \exp \left(i \lambda \sum_{k=1}^{N} \frac{a_{k} \phi_{n_{k}}(x)}{A_{N}}\right) d x, \\
N=1,2,3, \cdots,
\end{array}
$$

converge uniformly to $\Phi(\lambda: E)$ for $\lambda$ in any neighborhood $[\alpha, \beta]$ of the origin.

Let the system $\left\{\phi_{n}(x)\right\}$ be written as $\phi_{n}(x)=2 M \phi_{n}^{*}(x), n=1,2,3, \cdots$, whereupon the orthogonal system $\left\{\phi_{n}^{*}(x)\right\}$ has the uniform bound $1 / 2$. Observing that $e^{z}=(1+z) \exp \left(z^{2} / 2+Q(z)\right)$ is valid for $z \rightarrow 0$ with $Q(z)=o\left(z^{2}\right)$, we shall use Riesz products as in the paper of Salem and Zygmund [9]. Substituting $\left\{\phi_{n}^{*}(x)\right\}$ into (3.3) and applying the preceding formula $N$ times with $z_{N, k}=i 2 M \lambda a_{k} \phi_{n_{k}}^{*} / A_{N}$ we obtain

$$
\begin{aligned}
\Phi_{N}(\lambda: E)= & \frac{1}{m(E)} \int_{E} \exp \left(i 2 M \lambda \sum_{k=1}^{N} a_{k} \phi_{n_{k}}^{*}(x) / A_{N}\right) d x \\
= & \frac{1}{m(E)} \int_{E} \prod_{k=1}^{N}\left(1+i 2 M \lambda a_{k} \phi_{n_{k}}^{*}(x) / A_{N}\right) \\
& \cdot \exp \left(-(2 M)^{2} \lambda^{2} / 2 \sum_{k=1}^{N} a_{k}^{2} \phi_{n_{k}}^{* 2}(x) / A_{N}^{2}\right. \\
& \left.\quad+\sum_{k=1}^{N} Q\left(i 2 M \lambda a_{k} \phi_{n_{k}}^{*}(x) / A_{N}\right)\right) d x \quad(N=1,2,3, \cdots) .
\end{aligned}
$$

The proof proceeds with a number of lemmas the first of which is essentially Lemma 1 of Theorem I.

Lemma 1. For any sequence $\left\{a_{k}\right\}$ satisfying the conditions of the theorem,

$$
\frac{\max _{1 \leqq k \leqq N}\left(a_{k}\right)}{A_{N}} \rightarrow 0
$$$$
\text { as } N \rightarrow \infty \text {. }
$$

Returning to (3.4), it may be readily verified that since $z_{N, k} \rightarrow 0$ as $N \rightarrow \infty$ and $Q\left(z_{N, k}\right) / z_{N, k}^{4} \rightarrow 0$ as $z_{N, k} \rightarrow 0$,

$$
\exp \sum_{k=1}^{N} Q\left(z_{N, k}\right)=\exp o(1)
$$

uniformly for $x \in[a, b]$ and $\lambda$ on any finite range $[\alpha, \beta]$. Moreover, for all $x \in[a, b]$, all $\lambda \in[\alpha, \beta]$, and all $N$,

$$
\begin{aligned}
& \left|\prod_{k=1}^{N}\left(1+i 2 M \lambda a_{k} \phi_{n_{k}}^{*}(x) / A_{N}\right)\right|=\prod_{k=1}^{N}\left[1+(2 M)^{2} \lambda^{2} a_{k}^{2} \phi_{n_{k}}^{* 2}(x) / A_{N}^{2}\right]^{1 / 2} \\
& \leqq \exp \left(\lambda^{2} M^{2} / 2 \sum_{k=1}^{N} a_{k}^{2} / A_{N}^{2}\right)=\exp \left(\lambda^{2} M^{2} / 2\right)<C(\alpha, \beta) .
\end{aligned}
$$


The next lemma now follows easily.

Lemma 2. Define

$$
\begin{aligned}
\Phi_{N}^{*}(\lambda: E)= & \frac{1}{m(E)} \int_{E} \prod_{k=1}^{N}\left(1+i 2 M \lambda a_{k} \phi_{n_{k}}^{*}(x) / A_{N}\right) \\
& \cdot \exp \left(-(2 M)^{2} \lambda^{2} / 2 \sum_{k=1}^{N} a_{k}^{2} \phi_{n_{k}}^{* 2}(x) / A_{N}^{2}\right) d x .
\end{aligned}
$$

Then for any subsequence $\left\{\phi_{n_{k}}(x)\right\}$, any set $E \subset[a, b], m(E)>0$, and any sequence $\left\{a_{k}\right\}$ admissible in the sense of the theorem,

$$
\lim _{N \rightarrow \infty}\left|\Phi_{N}(\lambda: E)-\Phi_{N}^{*}(\lambda: E)\right|=0
$$

uniformly for $\lambda$ on any finite interval $[\alpha, \beta]$.

Proof. Irrespective of $\left\{\phi_{n_{k}}(x)\right\},\left\{a_{k}\right\}$, and $E$, the definitions of $\Phi_{N}$ and $\Phi_{N}^{*}$ together with (3.5) imply

$$
\left|\Phi_{N}(\lambda: E)-\Phi_{N}^{*}(\lambda: E)\right| \leqq \frac{C(\alpha, \beta)}{m(E)} \int_{a}^{b}\left|\exp \left(\sum_{k=1}^{N} Q\left(z_{N}, k\right)\right)-1\right| d x \leqq C(\alpha, \beta) \epsilon
$$

if $N$ is large.

The sequence of functions $\left\{\phi_{n}^{* 2}(x)\right\}$ and numbers $\left\{a_{k}^{2}\right\}$ may be considered as the functions $\left\{\psi_{n}(x)\right\}$ and sequence $\left\{\lambda_{k}\right\}$ of Theorem I. Hence, by Theorem I, there exists a subsequence $\left\{\phi_{n_{k}}^{2}(x)\right\}$ and function $g(x)$ in $L^{2}$ such that the sequence $\left\{\sum_{k=1}^{N} a_{k}^{2} \phi_{n_{k}}^{* 2}(x) / A_{N}^{2}\right\}$ converges in the $L^{2}$ mean to $g(x)$. Moreover, this choice of $\left\{\phi_{n_{k}}^{* 2}(x)\right\}$ and $g(x)$ is independent of the sequence $\left\{a_{k}^{2}\right\}$ satisfying the conditions of the theorem. Since a subsequence of the averages converges a.e. to $g(x)$ and since the averages are nonnegative and bounded by $1 / 4$, we may assume $0 \leqq g(x) \leqq 1 / 4, x \in[a, b]$. It also follows that $\int_{a}^{b} g(x) d x$ $=1 / 4$.

Suppose that the $\left\{\phi_{n_{k}}(x)\right\}$ corresponding to the $\left\{\phi_{n_{k}}^{*}(x)\right\}$ just selected had been used from the very beginning in defining $F_{N}(y: E)$ etc., and now define

$$
\begin{aligned}
\Phi_{N}^{* *}(\lambda: E)= & \frac{1}{m(E)} \int_{E} \prod_{k=1}^{N}\left(1+i 2 M \lambda a_{k} \phi_{n_{k}}^{*}(x) / A_{N}\right) \\
& \cdot \exp \left(-(2 M)^{2} \lambda^{2} g(x) / 2\right) d x, \quad N=1,2,3, \cdots .
\end{aligned}
$$

Lemma 3. For any set $E$ of positive measure and any admissible $\left\{a_{k}\right\}$, $\left[\Phi_{N}(\lambda: E)-\Phi_{N}^{* *}(\lambda: E)\right]$ tends to zero as $N \rightarrow \infty$ uniformly for $\lambda$ on any finite range $[\alpha, \beta]$.

Proof. By Lemma 2 it is enough to consider $\left[\Phi_{N}^{*}(\lambda: E)-\Phi_{N}^{* *}(\lambda: E)\right]$. 


$$
\left|\Phi_{N}^{*}(\lambda: E)-\Phi_{N}^{* *}(\lambda: E)\right|
$$

$$
\begin{aligned}
& \leqq \frac{C(\alpha, \beta)}{m(E)} \int_{E} \mid \exp \left(-(2 M)^{2} \lambda^{2} / 2 \sum_{k=1}^{N} a^{2} \phi_{n_{k}}^{* 2}(x) / A_{N}^{2}\right) \\
& -\exp \left(-(2 M)^{2} \lambda^{2} g(x) / 2\right) \mid d x .
\end{aligned}
$$

By the mean value theorem, $\left|e^{x_{1}}-e^{x_{2}}\right| \leqq K(\alpha, \beta)\left|x_{1}-x_{2}\right|$ for $x \in[a, b]$, $\lambda \in[\alpha, \beta]$ and where $x_{1}$ and $x_{2}$ are the exponents in (3.8). Thus

$$
\left|\Phi_{N}^{*}(\lambda: E)-\Phi_{N}^{* *}(\lambda: E)\right| \leqq \frac{(2 M)^{2} \bar{K}(\alpha, \beta)}{2 m(E)} \int_{a}^{b}\left|\sum_{k=1}^{N} a_{k}^{2} \phi_{n_{k}}^{* 2}(x) / A_{N}^{2}-g(x)\right| d x .
$$

But the integrals on the right tend to zero, proving the lemma.

The corollary to Theorem I shows that any subsequence of $\left\{\phi_{n_{k}}(x)\right\}$ may be used in the definitions of $F_{N}(y: E)$ and $\Phi_{N}^{* *}(\lambda: E)$, and Lemmas 2 and 3 would still be valid. To prove the present theorem it suffices therefore to show that $\left\{\phi_{n_{k}}(x)\right\}$ may be further refined to $\left\{\phi_{n_{k}}(x)\right\}$ independently of $\left\{a_{k}\right\}$ and set $E, m(E)>0$, so that using this refinement in definition of $F_{N}(y: E)$ and $\Phi_{N}^{* *}(\lambda: E)$ we have $\left[\Phi_{N}^{* *}(\lambda: E)-\Phi(\lambda: E)\right]$ tending to zero uniformly for $\lambda \in[\alpha, \beta]$, as $N \rightarrow \infty$, where in definition (3.2), $f(x)=4 M^{2} g(x)$. It is clear from the properties of $g(x)$ that $0 \leqq f(x) \leqq M^{2}, \int_{a}^{b} f(x) d x=1$.

Now for any $\lambda$,

$$
\begin{aligned}
& \left|\Phi_{N}^{* *}(\lambda: E)-\Phi(\lambda: E)\right| \\
& \quad=\left|\frac{1}{m(E)} \int_{E}\left[\prod_{k=1}^{N}\left(1+i 2 M \lambda a_{k} \phi_{n_{k}}^{*}(x) / A_{N}\right)-1\right] e^{-\lambda^{2}(2 M) 2 g(x) / 2}\right| d x .
\end{aligned}
$$

Using the series expansion for $e^{z}$ and integrating term-wise we obtain

$$
\begin{aligned}
\left|\Phi_{N}^{* *}(\lambda: E)-\Phi(\lambda: E)\right|= & \frac{1}{m(E)} \mid \sum_{j=0}^{\infty}(-1)^{j}\left(\lambda^{2} 2 M^{2}\right)^{j} / j ! \int_{E} g^{j}(x) \\
& \cdot\left[\prod_{k=1}^{N}\left(1+i 2 M \lambda a_{k} \phi_{n_{k}}^{*}(x) / A_{N}\right)-1\right] d x \mid \\
\leqq & \frac{1}{m(E)} \sum_{j=0}^{\infty}\left(2 M^{2} \lambda^{2}\right)^{j} / j ! \mid \int_{E} g^{j}(x) \\
& \cdot\left[\prod_{k=1}^{N}\left(1+i 2 M \lambda a_{k} \phi_{n_{k}}^{*}(x) / A_{N}\right)-1\right] d x \mid .
\end{aligned}
$$

LEMMA 4. Given any fixed set $E$, a subsequence $\left\{\phi_{n_{k}}(x)\right\}$ may be found such that for $\lambda$ on any finite interval $[\alpha, \beta]$ the corresponding $\Phi_{N}^{* *}(\lambda: E)$ tend uniformly 
to $\Phi(\lambda: E)$ irrespective of admissible sequences $\left\{a_{k}\right\}$. Moreover, any subsequence $\left\{\phi_{n_{k}}(x)\right\}$ of $\left\{\phi_{n_{k}}(x)\right\}$ also has this property.

Proof. Formula (3.10) shows that for uniform convergence, it is enough to demonstrate the existence of a subsequence $\left\{\phi_{n_{k}}(x)\right\}$ and constants $C_{N}(\alpha, \beta)$ for large $N$ such that $C_{N}(\alpha, \beta) \rightarrow 0$ as $N \rightarrow \infty$ and

$$
\left|\int_{E} g^{j}(x)\left[\prod_{k=1}^{N}\left(1+i 2 M \lambda a_{k} \phi_{n_{k}}^{*}(x) / A_{N}\right)-1\right] d x\right| \leqq C_{N}(\alpha, \beta)
$$

for every integer $j \geqq 0$ and every $\lambda \in[\alpha, \beta]$. For, granting this, the right side of (3.10) is majorized by

$$
\leqq \frac{C_{N}(\alpha, \beta)}{m(E)} \sum_{j=0}^{\infty} \frac{\left(2 M^{2} \lambda^{2}\right)^{j}}{j !}=\frac{C_{N}(\alpha, \beta)}{m(E)} e^{2 M^{2} \lambda^{2}} \rightarrow 0
$$

uniformly for $\lambda \in[\alpha, \beta]$, as $N \rightarrow \infty$.

Consider now the identity

$$
\begin{aligned}
\prod_{k=1}^{N}\left(1+a_{k}\right) \equiv\{1 & +\sum_{k=1}^{N} a_{k}+\sum_{1 \leqq i<j}^{N} a_{i} a_{j} \\
& \left.+\sum_{1 \leqq i<j<k}^{N} a_{i} a_{j} a_{k}+\cdots+\left(a_{1} a_{2} \cdots a_{N}\right)\right\}
\end{aligned}
$$

For each $j$ and every $N$ this formula establishes the inequality

$$
\begin{aligned}
& \left|\int_{E} g^{j}(x)\left[\prod_{k=1}^{N}\left(1+\frac{i 2 M \lambda a_{k} \phi_{n_{k}}^{*}(x)}{A_{N}}\right)-1\right] d x\right| \\
& \leqq \\
& \quad+\left|\frac{i 2 M \lambda}{A_{N}}\right| \sum_{k=1}^{N}\left|a_{k}\right|\left|\int_{E} g^{i}(x) \phi_{n_{k}}^{*}(x) d x\right| \\
& \quad+\left|\frac{i 2 M \lambda}{A_{N}}\right|^{2} \sum_{1 \leqq i<j}^{N}\left|a_{i}\right|\left|a_{j}\right|\left|\int_{E} g^{j}(x) \phi_{n_{i}}^{*}(x) \phi_{n_{j}}^{*}(x) d x\right| \\
& \quad+\cdots+\left|\frac{i 2 M \lambda}{A_{N}}\right|^{N}\left|a_{1}\right| \cdots\left|a_{i}\right|\left|a_{j}\right|\left|a_{k}\right|\left|\int_{E} g^{i}(x) \phi_{n_{i}}^{*}(x) \phi_{n_{j}}^{*}(x) \phi_{n_{k}}^{*}(x) d x\right|
\end{aligned}
$$

Now a number $N_{0}(\alpha, \beta)$ may be found by Lemma 1 so that for $N>N_{0}(\alpha, \beta)$,

$$
2 M|\lambda|\left(\max _{1 \leqq k \leqq N}\left|a_{k}\right|\right) / A_{N} \leqq 1 \quad \text { for } \lambda \in[\alpha, \beta]
$$

and therefore for $r=2,3,4, \cdots$ 


$$
(2 M)^{r}|\lambda| r\left(\max _{1 \leqq k \leqq N}\left|a_{k}\right|\right)^{r} / A_{N}^{r} \leqq 2 M|\lambda|\left(\max _{1 \leqq k \leqq N}\left|a_{k}\right|\right) / A_{N}
$$

for $\lambda$ on $[\alpha, \beta]$.

For such $N$, the right side of (3.13) is majorized by

$$
\begin{aligned}
& \leqq\left[2 M|\lambda|\left(\max _{1 \leqq k \leqq N}\left|a_{k}\right|\right) / A_{N}\right]\left[\sum_{k=1}^{N}\left|\int_{E} g^{j}(x) \phi_{n_{k}}^{*}(x) d x\right|\right. \\
& +\sum_{1 \leq i<j}^{N}\left|\int_{E} g^{j}(x) \phi_{n_{i}}^{*}(x) \phi_{n_{j}}^{*}(x) d x\right|+\cdots \\
& \left.+\left|\int_{E} g^{j}(x) \phi_{n_{1}}^{*}(x) \cdots \phi_{n_{N}}^{*}(x) d x\right|\right] \\
& \leqq\left[2 M(|\alpha|+|\beta|)\left(\max _{1 \leqq k \leqq N}\left|a_{k}\right|\right) / A_{N}\right] R_{N}(j)
\end{aligned}
$$

for all $\lambda$ on $[\alpha, \beta]$,

where $R_{N}(j)$ refers to the sums of integrals in the second bracket.

For each $j$ we divide the terms of the type appearing in $R_{N}(j)$ into groups of terms, defining the pth group as the collection of all terms

$$
\left|\int_{E} g^{j}(x) \phi_{n_{c}}^{*}(x) \phi_{n_{d}}^{*}(x) \cdots \phi_{n_{p}}^{*}(x) d x\right|
$$

in which $n_{p}$ is the largest subscript to appear. Then the terms which appear in $R_{N}(j)$ but not $R_{N-1}(j)$ constitute exactly the $N$ th group, and hence the $p$ th group does not increase with $N$.

We now note that the integrand in (3.15) is bounded, and so by the Riemann-Lebesgue property we may choose $\phi_{n_{k}}^{*}(x)$ so far out in the sequence $\left\{\phi_{n}^{*}(x)\right\}$ that a given term of type (3.15) is small. In fact, if $j$ is fixed, then for each $k, \phi_{n_{k}}^{*}(x)$ may be chosen so far out that

$$
\left[\begin{array}{l}
\text { sum of all terms in the } k \text { th } \\
\text { group of } R_{N}(j), N \geqq k
\end{array}\right] \leqq \frac{(b-a)}{2^{k}} .
$$

This implies that if the corresponding subsequence $\left\{\phi_{n_{k}}\right\}$ so determined were used to define $F_{N}(y: E)$ and $\Phi_{N}^{* *}(\lambda: E)$, we would have for this fixed $j$

(3.16) (a) $R_{\mathrm{v}}(j) \leqq(b-a)$, all $N$.

(b) If $\left\{\phi_{n_{k_{t}}}\right\}$, any subsequence of $\left\{\phi_{n_{k}}\right\}$, were used instead to define $\Phi_{N}^{* *}(\lambda: E)$, then $R_{N}(j)$ would still be exceeded by $(b-a)$ for the fixed $j$ under consideration since the terms in the $r$ th group of $R_{N}(j)$ relative to $\left\{\phi_{n_{k}}^{*}\right\}$ would be a subcollection of the terms in the $k_{r}$ th group of $R_{N}(j)$ relative to $\left\{\phi_{n_{k}}^{*}\right\}$ and hence have a sum not exceeding $(b-a) / 2^{k_{r}} \leqq(b-a) / 2^{r}$.

Employing the selection principle just described, we obtain a subsequence 
$\left\{\phi_{n_{k}}^{(0)}\right\}$ such that $R_{N}(0) \leqq(b-a)$, all $N$, if $\Phi_{N}^{* *}(\lambda: E)$ is defined starting with this subsequence. Pick a subsequence $\left\{\phi_{n_{k}}^{(1)}\right\}$ of $\left\{\phi_{n_{k}}^{(0)}\right\}$ by having the $p$ th element satisfy the following two conditions:

(a) $\left[\begin{array}{l}\text { sum of all terms in the } p \text { th group of } R_{N}(1) \text { (defined } \\ \text { with respect to the first }(\mathrm{p}-1) \text { elements of } \\ \left.\left\{\phi_{n_{k}}^{(1)}\right\}\right), N \geqq p\end{array}\right] \leqq \frac{(b-a)}{2^{p}}$,

(b) $\left[\begin{array}{l}\text { sum of all terms in the } p \text { th group of } R_{N}(1) \text { (defined } \\ \left.\text { with respect to } \phi_{n_{1}}^{(0)} \text { and } \phi_{n_{2}}^{(1)}, \phi_{n_{3}}^{(1)}, \cdots, \phi_{n_{p-1}}^{(1)}\right) \\ N \geqq p \geqq 2\end{array}\right] \leqq \frac{(b-a)}{2^{p}}$.

Then if $\Phi_{N}^{* *}(\lambda: E)$ is defined relative to $\left\{\phi_{n_{k}}^{(1)}\right\}$, we have $R_{N}(1) \leqq(b-a)$ for all $N$. If $\phi_{n_{1}}^{(1)}$ is replaced by $\phi_{n_{1}}^{(0)}$, then the sum of all groups of $R_{N}(1)$ beyond the first does not exceed $(b-a)$. In the same manner pick $\left\{\phi_{n_{k}}^{(2)}\right\},\left\{\phi_{n_{k}}^{(3)}\right\}, \cdots$, etc., each a subsequence of its predecessor and such that the $p$ th element of $\left\{\phi_{n_{k}}^{(j)}\right\}$ satisfies the conditions:

(a) $\left[\begin{array}{l}\text { sum of all terms in the } p \text { th group of } R_{N}(j)(\mathrm{de}- \\ \text { fined with respect to the first }(p-1) \text { elements of } \\ \left.\left\{\phi_{n_{k}}^{(j)}\right\}\right), N \geqq p\end{array}\right] \leqq \frac{(b-a)}{2^{p}}$,

(b) $\left[\begin{array}{l}\text { sum of all terms in the } p \text { th group of } R_{N}(j)(\mathrm{de}- \\ \text { fined with respect to } \phi_{n_{1}}^{(0)}, \phi_{n_{2}}^{(1)}, \phi_{n_{3}}^{(2)}, \cdots, \phi_{n_{j}}^{(j-1)}, \\ \left.\phi_{n_{j+1}}^{(j)}, \cdots, \phi_{n_{p-1}}^{(j)}\right), N \geqq p \geqq j+1\end{array}\right] \leqq \frac{(b-a)}{2^{p}}$.

Then if $\Phi_{N}^{* *}(\lambda: E)$ is defined relative to $\left\{\phi_{n_{k}}^{(\text {() }}\right\}$, we have $R_{N}(j) \leqq(b-a)$ for all $N$. If the first $j$ elements of $\left\{\phi_{n_{k}}^{(j)}\right\}$ are replaced by the $j$ diagonal elements from the preceding $j$ subsequences, the sum of the groups of $R_{N}(j)$, with the exception of the first $j$, will not exceed $(b-a) / 2^{j}$.

Finally, denote the diagonal subsequence of this collection of sequences by $\left\{\phi_{n_{k}}(x)\right\}$. If $\Phi_{N}^{* *}(\lambda: E)$ is defined with respect to this diagonal subsequence,

$$
R_{N}(j) \leqq(b-a)
$$

for each $j$ and all $N$.

This may be ascertained as follows. For each $j$ and all $N, R_{N}(j) \leqq(b-a) / 2^{j}$ + [the sum $Q_{j}$ of all terms in the first $j$ groups of $\left.R_{N}(j)\right] \leqq(b-a) / 2^{j}+Q_{j}$. This is because with the exception of the first $j$ terms, $\left\{\phi_{n_{k}}\right\}$ is a subsequence of $\left\{\phi_{n_{k}}^{(j)}\right\}$, and even with the first $j$ terms replaced by diagonal elements, the $k$ th group of $R_{N}(j), k>j$, has a sum not exceeding $(b-a) / 2^{k}$. The estimate for $Q_{j}$, on the other hand, depends on the fact that $Q_{j}$ contains as many terms of $R_{N}(j)$ as the number of nonvoid subsets of a set consisting of $j$-elements, i.e., $\left(2^{i}-1\right)$. Each element in $Q_{j}$ is of the form 


$$
S=\left|\int_{E} g^{j}(x) \phi_{n_{i_{1}}}^{*}(x) \cdots \phi_{n_{i_{r}}}^{*}(x) d x\right|
$$

where $1 \leqq r \leqq j$ and $n_{i_{1}}<n_{i_{2}} \leqq \cdots \leqq n_{i_{r}} \leqq n_{j}$. Then

$$
S \leqq(1 / 4)^{i}(1 / 2) m(E) \leqq(1 / 4)^{j} \cdot(1 / 2)(b-a) .
$$

Thus

$$
Q_{j} \leqq \frac{(b-a)\left(2^{j}-1\right)}{4^{i} \cdot 2} \leqq \frac{(b-a)}{2^{i+1}},
$$

and so

$$
R_{N}(j) \leqq \frac{(b-a)}{2^{j}}+\frac{(b-a)}{2^{j+1}},
$$

and (3.19) is demonstrated. Hence (3.14) shows that if we define

$$
C_{N}(\alpha, \beta)=\left[\frac{2 M[|\alpha|+|\beta|]\left(\max _{1 \leqq k \leqq N}\left|a_{k}\right|\right)}{A_{N}}\right](b-a)
$$

we shall have (3.11) for every $j \geqq 0$, and the lemma follows. We note that the final statement of the lemma is a consequence of the remark (3.16)-b. Lemma 4 is tantamount to proving the theorem for a particular fixed set $E, m(E)>0$.

Lемма 5. If $\left\{\phi_{n_{k}}(x)\right\}$ is the subsequence provided by Lemma 4 for the fixed set $E$ and for which $\left|\Phi_{N}(\lambda: E)-\Phi(\lambda: E)\right| \rightarrow 0$ uniformly for $\lambda$ on any finite interval, irrespective of admissible sequence $\left\{a_{k}\right\}$, we may replace any initial block of terms in $\left\{\phi_{n_{k}}(x)\right\}$ by other functions from the original orthonormal system, and we will still have this property. Any further refinement of the "new" subsequence will also have this property.

Proof. Suppose the first $n_{0}$ terms of $\left\{\phi_{n_{k}}(x)\right\}$ are replaced by $n_{0}$ other functions of our original orthonormal system, say $\phi_{n_{1}}, \phi_{n_{2}}, \cdots, \phi_{n_{n_{0}}}$. The function $\Phi_{N}^{* *}(\lambda: E)$ defined with the "new" functions will be denoted by

$$
\begin{aligned}
\tilde{\Phi}_{N}^{* *}(\lambda: E) & =\frac{1}{m(E)} \int_{E} \prod_{k=1}^{n_{0}}\left(1+\frac{i 2 M \lambda a_{k} \bar{\phi}_{n_{k}}^{*}(x)}{A_{N}}\right) \prod_{k=n_{0}+1}^{N}\left(1+\frac{i 2 M \lambda a_{k} \phi_{n_{k}}^{*}(x)}{A_{N}}\right) \\
& \cdot \exp \left(-(2 M)^{2} \lambda^{2} g(x) / 2\right) d x .
\end{aligned}
$$

Lemmas 2 and 3 are obviously valid for $\tilde{\Phi}_{N}^{* *}$ and the function $\tilde{\Phi}_{N}(\lambda: E)$ of (3.4) (defined here with the "new" subsequence) since the proof of the Banach-Saks result, Theorem I, is not changed if we alter an initial finitely long block of terms. If we wish to show that $\left|\tilde{\Phi}_{N}(\lambda: E)-\Phi(\lambda: E)\right| \rightarrow 0$ uniformly for $\lambda=O(1)$, it is therefore enough to show that $\left|\tilde{\Phi}_{N}^{* *}(\lambda: E)-\Phi(\lambda: E)\right|$ $\rightarrow 0$ uniformly for $\lambda$ on any finite range. This will be done if we show that 
$\left|\tilde{\Phi}_{N}^{* *}(\lambda: E)-\Phi_{N}^{* *}(\lambda: E)\right| \rightarrow 0$ uniformly for $\lambda$ on a finite range, where $\Phi_{N}^{* *}$ is defined with the subsequence $\left\{\phi_{n_{k}}\right\}$ provided by Lemma 4 . Now

$$
\begin{aligned}
m(E) \mid \Phi_{N}^{* *}(\lambda: E)- & \tilde{\Phi}_{N}^{* *}(\lambda: E) \mid \\
=\mid & \int_{E}\left[\prod_{k=1}^{n_{0}}\left(1+\frac{i 2 M \lambda a_{k} \phi_{n_{k}}^{*}}{A_{N}}\right)-\prod_{k=1}^{n_{0}}\left(1+\frac{\left.\left.i 2 M \lambda a_{k}{\boldsymbol{\phi}_{n_{k}}^{*}}_{A_{N}}^{*}\right)\right]}{A_{k=n_{0}+1}}\left(1+\frac{i 2 M \lambda a_{k} \phi_{n_{k}}^{*}(x)}{A_{N}}\right) \exp \left(-(2 M)^{2} \lambda^{2} g(x) / 2\right) d x \mid,\right.\right.
\end{aligned}
$$

and applying (3.5),

$$
\leqq \bar{C}(\alpha, \beta) \int_{E}\left|\prod_{k=1}^{n_{0}}\left(1+\frac{i 2 M \lambda a_{k} \phi_{n_{k}}^{*}}{A_{N}}\right)-\prod_{1}^{n_{0}}\left(1+\frac{i 2 M \lambda a_{k} \bar{\phi}_{n_{k}}^{*}}{A_{N}}\right)\right| d x .
$$

But since $n_{0}$ is fixed, the integrand is clearly bounded and tends to zero as $N \rightarrow \infty$ because $A_{N} \rightarrow \infty$. The integral then tends to zero so that the result is established. The last statement of the lemma is obvious.

Let $I_{r}, r=1,2,3, \cdots$, be an enumeration of all rational subintervals of $[a, b]$. We shall now show that there exists a master-subsequence $\left\{\phi_{n_{k}}(x)\right\}$ of the given orthonormal sequence $\left\{\phi_{n}(x)\right\}$ such that if, for each rational subinterval $I_{r}$ of $[a, b]$, we define $F_{N}\left(y: I_{r}\right)$ and $\Phi_{N}^{* *}\left(\lambda: I_{r}\right)$ with this subinterval and this subsequence, $\Phi_{N}^{* *}\left(\lambda: I_{r}\right)$ will converge uniformly for $\lambda$ on any finite interval $[\alpha, \beta]$ and any admissible sequence $\left\{a_{k}\right\}$ to $\Phi\left(\lambda: I_{r}\right), r=1,2,3, \cdots$. Any subsequence of $\left\{\phi_{n_{k}}(x)\right\}$ enjoys the same property.

To obtain such a master subsequence, we employ Lemma 4 with $E \equiv I_{1}$ and find a subsequence $\left\{\phi_{n}^{(1)}\right\}$ such that for it or any subsequence of it the corresponding $\left\{\Phi_{N}^{* *}\left(\lambda: I_{1}\right)\right\}$ converges uniformly to $\Phi\left(\lambda: I_{1}\right), \lambda=O(1)$. With $E \equiv I_{2}$, Lemma 4 tells how to find a subsequence $\left\{\phi_{n}^{(2)}\right\}$ of $\left\{\phi_{n}^{(1)}\right\}$ such that for it or any subsequence of it the corresponding $\left\{\Phi_{N}^{* *}\left(\lambda: I_{2}\right)\right\}$ converges uniformly to $\Phi\left(\lambda: I_{2}\right), \lambda=O(1)$. Similarly we determine subsequences $\left\{\phi_{n}^{(3)}\right\}$, $\left\{\phi_{n}^{(\mathbf{(})}\right\}, \cdots$, etc., each a subsequence of its predecessor and with $\left\{\phi_{n}^{(t)}\right\}$ having the property that for it or any subsequence of it, the corresponding $\left\{\Phi_{N}^{* *}\left(\lambda: I_{t}\right)\right\}$ converges uniformly to $\Phi\left(\lambda: I_{t}\right), \lambda=O(1)$. Now take the diagonal subsequence of the subsequences $\left\{\phi_{n}^{(t)}\right\}, t=1,2,3, \cdots$, and denote it by $\left\{\phi_{n_{k}}(x)\right\}$. Define $F_{N}\left(y: I_{r}\right)$ and $\Phi_{N}^{* *}\left(\lambda: I_{r}\right)$ by means of this master subsequence, $r=1,2,3, \cdots$. It may then be asserted that for this final subsequence $\Phi_{N}^{* *}\left(\lambda: I_{t}\right) \rightarrow \Phi\left(\lambda: I_{t}\right)$ for $t=1,2,3, \cdots$, uniformly for $\lambda$ on any finite range. This follows from Lemma 5 , and the observation that for each $t \geqq 1$, $\left\{\phi_{n_{k}}(x)\right\}$ is a subsequence of $\left\{\phi_{n_{k}}^{(t)}(x)\right\}$, if we alter the first $t$ terms. We have therefore proved the theorem for every rational subinterval of $[a, b]$.

LEMмA 6. If $F_{N}(y: E)$ and $\Phi_{N}^{* *}(\lambda: E)$ are defined with the master subsequence 
$\left\{\phi_{n_{k}}\right\}$ (or any refinement of $i t$ ) and any measurable set $E \subset[a, b], m(E)>0$, then $\Phi_{N}^{* *}(\lambda: E)$ will converge uniformly for $\lambda$ on any finite range to $\Phi(\lambda: E)$ as $N \rightarrow \infty$ independently of admissible sequence $\left\{a_{k}\right\}$.

Proof. It is enough to prove the lemma for Borel sets of positive measure. Given any $\epsilon>0$ and any Borel set $E$, there is a finite union of disjoint rational interval $E_{0}=\bigcup_{i-1}^{q} I_{r_{i}}$ such that

$$
\left|E-E_{0}\right|<\epsilon, \quad\left|E_{0}-E\right|<\epsilon .
$$

Also for any integrable $f(x)$ we have

$$
\int_{E} f(x) d x=\int_{E_{0}} f(x)+\int_{\left(E-E_{0}\right)} f d x-\int_{\left(E_{0}-E\right)} f d x
$$

Apply this to the expression

$$
\begin{aligned}
m(E) \mid & \Phi_{N}^{* *}(\lambda: E)-\Phi(\lambda: E) \mid \\
= & \mid \int_{E} \prod_{j=1}^{N}\left(1+i 2 M \lambda a_{j} \phi_{n_{k_{j}}}^{*} / A_{N}\right) \exp \left(-\lambda^{2} f(x) / 2\right) \\
& \quad-\int_{E} \exp \left(-\lambda^{2} f(x) / 2\right) d x \mid .
\end{aligned}
$$

With $\int$ referring to the first integral on the right in (3.23) and $\int^{\prime}$ to the second, (3.22) implies:

$$
\begin{aligned}
& \leqq\left|\int_{E_{0}}-\int_{E_{0}}^{\prime}\right|+\left|\int_{\left(E-E_{0}\right)}-\int_{E-E_{0}}^{\prime}\right|+\left|\int_{\left(E_{0}-E\right)}-\int_{\left(E_{0}-E\right)}^{\prime}\right| \\
& \leqq \sum_{i=1}^{q}\left|\int_{I_{r_{i}}}-\int_{I_{r_{i}}}^{\prime}\right|+\left|\int_{\left(E-E_{0}\right)}-\int_{\left(E-E_{0}\right)}^{\prime}\right|+\left|\int_{\left(E_{0}-E\right)}-\int_{\left(E_{0}-E\right)}^{\prime}\right| \\
& =D_{1}+D_{2}+D_{3} .
\end{aligned}
$$

Now independently of $\left\{a_{k}\right\}$, each integral belonging to $D_{1}$ is less than $\epsilon / q$ uniformly for $\lambda \in[\alpha, \beta]$ if $N>N_{0}(\epsilon)$, by the discussion preceding Lemma 6 . In both $D_{2}$ and $D_{3}$ the integrand is bounded uniformly in $N$ for $\lambda \in[\alpha, \beta]$ and $x \in[a, b]$, say by $C(\alpha, \beta)$, and since the measures of the range of integration are in each case less than $\epsilon$, the estimate for (3.23) is

This proves Lemma 6.

$$
\leqq \epsilon+2 C(\alpha, \beta) \epsilon \text {. }
$$

The theorem is also proved, since throughout the discussion $[\alpha, \beta]$ referred only to an arbitrary interval and the choice of $\left\{\phi_{n_{k}}(x)\right\}$, the master subsequence, was independent of $[\alpha, \beta]$. By repeating the proof of the corollary to Theorem I, or even by direct inspection of Theorem II, we can prove:

CoROllaRy. For any subsequence $\left\{\phi_{n_{k_{j}}}(x)\right\}$ of the subsequence provided by Theorem II, the distribution functions $F_{N}(y: E)$ defined with this new subsequence 
will have the properties asserted in Theorem II with respect to the same function $f(x)$ appearing in the definition of $\Phi(\lambda: E)$.

Remarks. (1) The "pseudo-Gaussian" form of the characteristic function of the limiting distribution, that is,

$$
\Phi(\lambda: E)=\frac{1}{m(E)} \int_{E} \exp \left(-\lambda^{2} f(x) / 2\right) d x, \quad 0 \leqq f(x) \leqq M^{2},
$$

is in best possible form. We could not expect $f(x)$ to be a constant. Consider, for example, the Walsh system( $\left(^{3}\right)$ defined on $[0,1]$. If a Walsh system $\left\{\psi_{k}^{(1)}(x)\right\}$ is constructed on $[0,1)$ and another $\left\{\psi_{k}^{(2)}(x)\right\}$ on $[1,2]$, and if we define

$$
\rho_{k}(x)=\left\{\begin{array}{lll}
(1 / 2) \psi_{k}^{(1)}(x) & \text { on } & {[0,1),} \\
\left(3^{1 / 2} / 2\right) \psi_{k}^{(2)}(x) & \text { on } & {[1,2],}
\end{array} \quad k=1,2,3, \cdots,\right.
$$

we find that $\left\{\rho_{k}(x)\right\}$ is an orthonormal system on $[0,2]$ with uniform bound $M=3^{1 / 2} / 2$.

Consider the revised system $\rho_{k}^{*}(x)=\rho_{k}(x) / 3^{1 / 2}, k=1,2,3, \cdots$ as is used in Theorem III. If Theorem I is applied to the functions $\left\{\rho_{\boldsymbol{k}}^{* 2}(x)\right\}$ to obtain a limiting function $g(x)$, the identities

$$
\sum_{k=1}^{N} \frac{a_{k \rho}^{2} \rho_{n_{k}}^{* 2}(x)}{A_{N}^{2}} \equiv t(x)=\left\{\begin{array}{lll}
1 / 12 & \text { on } & {[0,1),} \\
1 / 4 & \text { on } & {[1,2],}
\end{array} \quad N=1,2,3, \cdots,\right.
$$

$\left\{\rho_{n_{k}}^{* 2}(x)\right\}$ any subsequence of $\left\{\rho_{k}^{* 2}(x)\right\}$, imply that the only limiting distribution $\Phi(\lambda: E)$ which could be obtained by the techniques of Theorem II in the case $E=[0,2]$ is

$$
\Phi(\lambda:[0,2])=\frac{1}{2} \int_{0}^{2} \exp \left(-3 \lambda^{2} t(x) / 2\right) d x=\frac{\exp \left(-\lambda^{2} / 8\right)+\exp \left(-3 \lambda^{2} / 8\right)}{2} .
$$

(2) We may consider Theorem III as establishing a mapping between uniformly bounded, real-valued, orthonormal systems, and nonnegative, bounded functions. This mapping is not unique even under the additional assumption of completeness. Consider, for example, a Walsh system $\left\{\psi_{k}^{(1)}(x)\right\}$ on $[0,1]$ which is considered to be on $[0,2]$ by defining each function as zero on $(1,2]$. Consider a similar Walsh system $\left\{\psi_{\boldsymbol{k}}^{(2)}(x)\right\}$ on $[1,2]$ which is 0 on $[0,1)$. Form an orthonormal system $\left\{\phi_{n}(x)\right\}$ which alternates functions of

(3) For a discussion of the Walsh system see N. J. Fine [3]. These functions are block functions defined on $[0,1]$ as follows: $\psi_{0}(x) \equiv 1, \psi_{N}(x)=\prod_{i=1}^{r} \phi_{m_{i}}(x)$ where $N=\sum_{i=1}^{r} 2^{m_{i}}$ and the $\phi$ 's are Rademacher functions, $\phi_{0}(x)=1(0 \leqq x<1 / 2), \phi_{0}(x)=-1(1 / 2 \leqq x<1), \phi_{0}(x+1)=\phi_{0}(x)$, $\phi_{n}(x)=\phi_{0}\left(2^{n} x\right)$. 
the two given sequences, i.e., $\left\{\psi_{1}^{(1)}(x), \psi_{1}^{(2)}(x), \psi_{2}^{(1)}(x), \psi_{2}^{(2)}(x), \cdots\right\}$. This system is complete and uniformly bounded. It is clearly possible, however, under Theorem I, to get two distinct limit functions $g(x)$ for the modified system $\left\{\phi_{n}^{*}(x)=\phi_{n}(x) / 2\right\}$, and hence two different $f(x)=4 g(x)$ depending on which subsequence, $\left\{\psi_{k}^{(1)}(x)\right\}$ or $\left\{\psi_{k}^{(2)}(x)\right\}$, is chosen to represent the combined sequence.

Consideration of the proof of Theorem II will lead to the further conclusion that the determination of conditions under which the mapping is unique is equivalent to determining when, for a uniformly bounded orthonormal sequence $\left\{\phi_{n}(x)\right\}$, the sequence of squared functions $\left\{\phi_{n}^{2}(x)\right\}$ will have a single weak limit for all possible subsequences. Despite this nonuniqueness, the next theorem shows that to within a constant, every bounded, nonnegative function for which $\int_{a}^{b} f(x) d x \neq 0$ is an image under the mapping. The conditional phrase, "to within a constant," appears in the theorem when we normalize such an $f(x)$ by $\left(\int_{a}^{b} f(x) d x\right)^{-1}$. The theorem is stated for the interval $[0,1]$, but the proof obviously generalizes.

THEOREM III. Given any bounded, nonnegative function $f(x)$ on $[0,1]$, of nonzero mean value, there exists a uniformly bounded, real-valued, orthonormal system $\left\{\phi_{k}(x)\right\}$ on $[0,1]$ such that for any real sequence $\left\{a_{k}\right\}$ satisfying

(a) $A_{N}=\left(a_{1}^{2}+a_{2}^{2}+\cdots+a_{N}^{2}\right)^{1 / 2} \rightarrow \infty$, as $N \rightarrow \infty$,

(b) $a_{N}=o\left(A_{N}\right)$

and any set $E, m(E)>0$, the distribution functions

$$
F_{N}(y: E)=\frac{m\left(\left\{x \in E \mid \sum_{k=1}^{N} a_{k} \phi_{k}(x) / A_{N} \leqq y\right\}\right)}{m(E)}, \quad N=1,2,3, \cdots
$$

tend to a limiting distribution $F(y: E)$ whose characteristic function is

$$
\Phi(\lambda: E)=\frac{1}{m(E)} \int_{E} \exp \left(-c \lambda^{2} f(x) / 2\right) d x,
$$

where $c=\left\{\int_{0}^{1} f(x) d x\right\}^{-1}$, a normalizing factor.

Proof. A scrutiny of the techniques in the proof of Theorem II will suffice to show that we need only to obtain an orthonormal, uniformly bounded, realvalued system on $[0,1]$, such that for it and any of its subsequences $\left\{\phi_{n_{k}}(x)\right\}$ we have

$$
\frac{\sum_{1}^{N} a_{k}^{2} \phi_{n_{k}}^{2}(x)}{c A_{N}^{2}} \rightarrow f(x)
$$

in the mean on $[0,1]$ independently of $\left\{a_{k}\right\}$, where $f(x)$ is the given function and $c$ is the normalizing constant for $f(x)$. If this is done, the final subsequence 
obtained by the methods of Theorem II may be designated as $\left\{\phi_{k}(x)\right\}$ in the above theorem and the result will be established.

Consider the Walsh-Fourier series $\sum_{k=0}^{\infty} c_{k} \psi_{k}(x)$ for $f(x)$; that is, $c_{k}$ $=\int_{0}^{1} f(x) \psi_{k}(x) d x$. It is known (see Paley [7]) that the $2^{n}$ th partial sums, $S_{2}{ }^{n}(x)$ $=\sum_{\boldsymbol{k}=0}^{2^{n}-1} c_{k} \psi_{k}(x)$, converge a.e. to $f(x)$. It is also well known that we may write $S_{2^{n}}(x)=\int_{0}^{1} f(x+t) D_{2^{n}}(t) d t$ where $D_{2^{n}}(t)=\sum_{\substack{k=0 \\\left(2^{n}-1\right)}} \psi_{k}(t)$ is the $2^{n}$ th Dirichlet kernel and " $\dot{+}$ " is a measure preserving transformation defined by Fine in his study of the Walsh system $\left(^{4}\right)$. The kernel is nonnegative and $\int_{0}^{1} D_{2^{n}}(t) d t=1$, all $n$. These facts imply that $S_{2^{n}}(x)$ is nonnegative and bounded by the bound of $f(x)$ uniformly in $n$ and $x$. As a consequence, $S_{2^{n}}(x)$ converges to $f(x)$ in the $L^{1}$ metric on $[0,1]$.

We further know from the properties of the Walsh functions that the $S_{2^{n}}(x)$ are really step functions with points $p_{m}=m / 2^{n}, 1 \leqq m \leqq 2^{n}$, as points of jump and intervals of constancy of length $1 / 2^{n}$. Therefore we define

$$
\rho_{n}(x)=c^{1 / 2} \psi_{2^{n}}(x)\left(S_{2^{n}}(x)\right)^{1 / 2}
$$

for each $n$ where $\psi_{2^{n}}(x)$ is the $2^{n}$ th Walsh function and $c=\left(\int_{0}^{1} f(x) d x\right)^{-1}>0$. Now

$$
\int_{0}^{1} \rho_{n}^{2}(x) d x=\frac{\int_{0}^{1} S_{2^{n}}(x) d x}{\int_{0}^{1} f(x) d x}=\frac{\int_{0}^{1}\left(\sum_{k=0}^{2^{n}-1} c_{k} \psi_{k}(x)\right) d x}{\int_{0}^{1} f(x) d x}=\frac{c_{0}}{\int_{0}^{1} f(x) d x}=1
$$

for each $n \geqq 1$.

It is here that the normalizing factor is needed. Also

$$
\int_{0}^{1} \rho_{n}(x) \rho_{m}(x) d x=0
$$$$
n \neq m,
$$

since if $n+1 \leqq m$, every interval of constancy of $\rho_{n}$ is of the form $\left[p / 2^{n+1}\right.$, $p+1 / 2^{n+1}$. Divide each such interval into subintervals of length $1 / 2^{m}$. On each such, $\int \rho_{m}(x) d x$ is zero because $S_{2^{m}}(x)$ is constant while $\psi_{2^{m}}(x)$ is +1 on the first half of the subinterval and -1 on the last half. Thus $\left\{\rho_{n}\right\}$ is a uniformly bounded, orthonormal system. Now for any subsequence $\left\{\rho_{n_{k}}(x)\right\}$, we have

$$
\frac{\sum_{k=1}^{N} a_{k}^{2} \rho_{n_{k}}^{2}(x)}{c A_{N}^{2}}=\frac{\sum_{k=1}^{N} a_{k}^{2} S_{2^{n_{k}}}(x)}{A_{N}^{2}},
$$

(4) If $x=\sum_{i=1}^{\infty} x_{i} / 2^{i}$ and $y=\sum_{i=1}^{\infty} y_{i} / 2^{i}$ are the dyadic representations of $x$ and $y, 0 \leqq x$, $y \leqq 1$, then $x+y=\sum_{i=0}^{\infty}\left|x_{i}-y_{i}\right| / 2^{i}$. 
and clearly this converges boundedly a.e. (and hence converges in the $L^{1}$ metric) to $f(x)$, and the theorem is proved.

We end this section with a result of the type obtained by Kac (see (1.3)). The proof follows the general pattern of the proof of Theorem II except that a substitute technique must be found at the point where the RiemannLebesgue theorem was employed. Because of the similarity to Theorem II only a sketch of the proof will be given.

THEOREM IV. Let $f(x)$ be bounded on $[a, b]$, of period $(b-a)$, and $\int_{a}^{b} f(x) d x=0$, $\int_{a}^{b} f^{2}(x) d x \neq 0$. Then there exists a subsequence $\left\{f\left(n_{k} x\right)\right\}$ of the sequence of functions $\{f(n x)\}, n=1,2,3, \cdots$, such that for any sequence of real numbers $\left\{a_{k}\right\}$ satisfying

(a) $A_{N}=\left(a_{1}^{2}+a_{2}^{2}+\cdots+a_{N}^{2}\right)^{1 / 2} \rightarrow \infty$, as $N \rightarrow \infty$,

(b) $a_{N}=o\left(A_{N}\right)$

and any measurable set $E \subset[a, b], m(E)>0$, the distribution functions

$$
F_{N}(y: E)=\frac{m\left(\left\{x \in E \mid \sum_{k=1}^{N} \frac{a_{k} f\left(n_{k} x\right)}{A_{N}} \leqq y\right\}\right)}{m(E)}, \quad N=1,2,3, \cdots,
$$

converge to the Gaussian distribution with mean value zero and standard deviation $=\left(\int_{a}^{b} f^{2}(x) d x\right)^{1 / 2}$.

Proof. It is sufficient to show the existence of a subsequence $\left\{f\left(n_{k} x\right)\right\}$ such that independently of $\left\{a_{k}\right\}$ and subset $E \subset[a, b]$ the characteristic functions for the $F_{N}(y: E)$ defined with the subsequence $\left\{f\left(n_{k} x\right)\right\}$,

$$
\Phi_{N}(\lambda: E)=\int_{-\infty}^{\infty} e^{i \lambda y} d F_{N}(y: E),
$$

converge uniformly for $\lambda$ on any finite range to the Gaussian characteristic function

$$
\Phi(\lambda)=\exp \left(-\lambda^{2} \sigma^{2} / 2\right) \quad \text { where } \sigma=\left(\int_{0}^{1} f^{2}(x) d x\right)^{1 / 2} .
$$

The early techniques of the proof of Theorem II and also Lemma 2 of that proof will hold here so that it is enough to show the uniform convergence for $\lambda$ on any finite range of

$$
\Phi_{N}^{*}(\lambda: E)=\frac{1}{m(E)} \int_{E} \exp \left(-\lambda^{2} / 2 \sum_{k=1}^{N} a_{k}^{2} f^{2}\left(n_{k} x\right) / A_{N}^{2}\right) \prod_{k=1}^{N}\left[1+\frac{i \lambda a_{k} f\left(n_{k} x\right)}{A_{N}}\right] d x
$$

to $\Phi(\lambda)$. The first lemma is well known (cf. Zygmund [10]).

Lemma 1. If $f(x) \in L(a, b)$ and $h(x)$ is bounded, both periodic, then

$$
\lim _{n \rightarrow \infty} \int_{a}^{b} f(x) h(n x) d x=\left(\int_{a}^{b} f(x) d x\right)\left(\int_{a}^{b} h(x) d x\right) .
$$


LEMma 2. From the sequence $\{f(n x)\}$ of the theorem can be extracted a subsequence $\left\{f\left(n_{k} x\right)\right\}, k=1,2, \cdots$, such that for any sequence of real numbers $\left\{a_{k}\right\}$ admissible in the sense of the theorem

$$
\frac{\sum_{k=1}^{N} a_{k}^{2} f^{2}\left(n_{k} x\right)}{A_{N}^{2}} \rightarrow\left(\int_{a}^{b} f^{2}(x) d x\right)=\sigma^{2} \text { in the } L^{2} \text { mean on }[a, b] .
$$

Moreover, any subsequence $\left\{f^{2}\left(n_{k_{j}} x\right)\right\}$ of $\left\{f^{2}\left(n_{k} x\right)\right\}$ also has this property.

Proof. By Lemma 1,

$$
\int_{a}^{b} p(x) f^{2}(n x) d x \rightarrow\left(\int_{a}^{b} p(x) d x\right)\left(\int_{a}^{b} f^{2}(x) d x\right)=\int_{a}^{b} p(x) \cdot \sigma^{2} d x
$$

for every periodic $p(x)$ in $L^{2}(a, b)$. This implies $\left\{f^{2}(n x)\right\}$ converges weakly to the constant $\sigma^{2}$, and hence, by Theorem I, there exists a subsequence $\left\{f^{2}\left(n_{k} x\right)\right\}$, such that for any admissible $\left\{a_{k}\right\}$,

$$
\frac{\sum_{k=1}^{N} a_{k}^{2} f^{2}\left(n_{k} x\right)}{A_{N}^{2}} \rightarrow \sigma^{2} \text { in } L^{2} \text { mean. }
$$

This proves the lemma. The last statement in the lemma follows from the corollary of Theorem I.

Using this subsequence $\left\{f^{2}\left(n_{k} x\right)\right\}$ and arguments paralleling those of Lemma 3, Theorem II, we find that for any $E, m(E)>0$, the function

$$
\begin{aligned}
\Phi_{N}^{* *}(\lambda: E) & =\frac{1}{m(E)} \int_{E} \prod_{k=1}^{N}\left(1+\frac{i \lambda a_{k} f\left(n_{k} x\right)}{A_{N}}\right) \exp \left(-\lambda^{2} \sigma^{2} / 2\right) d x \\
& =\frac{\exp \left(-\lambda^{2} \sigma^{2} / 2\right)}{m(E)} \int_{E} \prod_{k=1}^{N}\left(1+\frac{i \lambda a_{k} f\left(n_{k} x\right)}{A_{N}}\right) d x
\end{aligned}
$$

differs from $\Phi_{N}^{*}(\lambda: E)$ by an error tending uniformly to zero for $\lambda$ on any finite range, independently of $\left\{a_{k}\right\}$.

It is therefore sufficient to refine $\left\{f\left(n_{k} x\right)\right\}$ to $\left\{f\left(n_{k_{j}} x\right)\right\}$ such that if $\Phi_{N}^{* *}(\lambda: E)$ is defined with this subsequence, $\Phi_{N}^{* *}(\lambda: E)$ tends to $\Phi(\lambda)$ uniformly for $\lambda$ on any finite range independently of $\left\{a_{k}\right\}$ and set $E$. From (3.25) this will be accomplished if it can be shown that

$$
I_{N}(\lambda: E)=\int_{E} \prod_{j=1}^{N}\left(1+\frac{i \lambda a_{j} f\left(n_{k_{j}} x\right)}{A_{N}}\right) d x
$$

tends to $m(E)$ uniformly for $\lambda$ on a finite range and independently of set $E$ and $\left\{a_{k}\right\}$. 
Expanding again by the formula (3.12) of Theorem II,

$$
\begin{aligned}
& \left|I_{N}(\lambda: E)-m(E)\right| \\
& \leqq\left|\frac{i \lambda}{A_{N}}\right| \sum_{j=1}^{N}\left|a_{j}\right|\left|\int_{E} f\left(n_{k_{j}} x\right) d x\right|+\left|\frac{i \lambda}{A_{N}}\right|^{2} \sum_{1 \leqq i<j}^{N}\left|a_{i}\right|\left|a_{j}\right|\left|\int_{E} f\left(n_{k_{i}} x\right) f\left(n_{k_{j}} x\right) d x\right| \\
& \quad+\left|\frac{i \lambda}{A_{N}}\right|^{3} \sum_{1 \leqq i<j<t}^{N}\left|a_{i}\right|\left|a_{j}\right|\left|a_{t}\right|\left|\int_{E} f\left(n_{k_{i}} x\right) f\left(n_{k_{j}} x\right) f\left(n_{k_{t}} x\right) d x\right|+\cdots \\
& \quad+\left|\frac{i \lambda}{A_{N}}\right|^{N}\left|a_{1}\right| \cdots\left|a_{N}\right|\left|\int_{E} f\left(n_{k_{i}} x\right) \cdots f\left(n_{k_{N}} x\right) d x\right| \\
& \leqq
\end{aligned}
$$

where $R_{N}(E)$ is defined as in Theorem II. The integrals in $R_{N}(E)$ are of the form

$$
\left|\int_{a}^{b} \chi_{E}(x) f\left(n_{c} x\right) f\left(n_{d} x\right) \cdots f\left(n_{p} x\right) d x\right|
$$

and by Lemma 1 can be made small in absolute value by choosing $n_{p}$ sufficiently large. This is the key to the proof and serves as did the RiemannLebesgue property in Theorem II.

Now enumerate the rational subintervals of $[a, b]$ and with $E$ replaced by $J_{r}$, a typical rational subinterval, refine subsequence $\left\{f\left(n_{k} x\right)\right\}$ to $\left\{f^{(r)}\left(n_{k} x\right)\right\}$, as in Theorem II so as to make $I_{N}\left(\lambda: J_{r}\right)$ tend to $m\left(J_{r}\right)$ uniformly for $\lambda$ on a finite range. The diagonal subsequence of the subsequences $\left\{f^{(r)}\left(n_{k} x\right)\right\}$, $r=1,2,3, \cdots$, is such that if the distribution functions $F_{N}(\lambda: E)$ are defined with it, these distributions will converge for $E=J_{r}, r=1,2,3, \cdots$.

The extension to arbitrary sets $E$ of positive measure is done exactly as in the last lemma of Theorem II.

4. Complex-valued systems. Let $\left\{\theta_{n}(x)\right\}$ be a uniformly bounded, complex-valued, orthonormal system on a finite interval $[a, b]$ with bound $M$. Each $\theta_{n}(x)$ may be decomposed, $\theta_{n}(x)=r_{n}(x)+i s_{n}(x)$, where $r_{n}(x)$ and $s_{n}(x)$ are real-valued. Therefore the given orthonormal system gives rise to two sequences of random variables, $\left\{r_{n}(x)\right\}$ and $\left\{s_{n}(x)\right\}$. Moreover, for any subsequence of these, $\left\{r_{n_{k}}(x)\right\},\left\{s_{n_{k}}(x)\right\}$, the functions

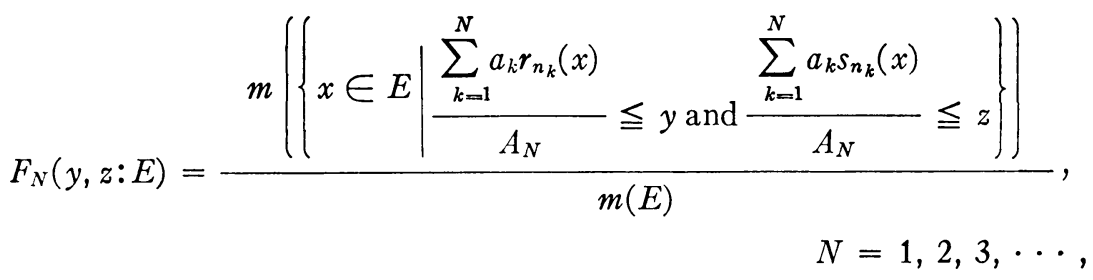


represent for each $N$ a two dimensional distribution of unit mass over the plane. Here $\left\{a_{k}\right\}$ is any real sequence satisfying

$$
\begin{aligned}
& \text { (a) } A_{N}=\left(a_{1}^{2}+a_{2}^{2}+\cdots+a_{N}^{2}\right)^{1 / 2} \rightarrow \infty \quad \text { as } N \rightarrow \infty \text {, } \\
& \text { (b) } a_{N}=o\left(A_{N}\right)
\end{aligned}
$$

and $E \subset[a, b]$ has positive measure.

The sequence $\left\{F_{N}(y, z: E)\right\}$ will converge to a limiting distribution if we show that for any rectangle $R$ in the $\lambda \mu$ plane the characteristic functions of the $F_{N}$,

$$
\Phi_{N}(\lambda, \mu: E)=\int_{-\infty}^{\infty} \int_{-\infty}^{\infty} \exp (i(\lambda y+\mu z)) d F_{N}(y, z: E)
$$

tend uniformly to a limit function $\Phi(\lambda, \mu: E)$.

Defining $r_{n}^{*}(x)=r_{n}(x) / 2 M, s_{n}^{*}(x)=s_{n}(x) / 2 M$, we have $\left|r_{n}^{*}(x)\right| \leqq 1 / 2$, $\left|s_{n}^{*}(x)\right| \leqq 1 / 2$ for each $n$. Also,

$$
\begin{aligned}
\Phi_{N}(\lambda, \mu: E)= & m(E)^{-1} \int_{E} \exp \left(i 2 M \left(\lambda \sum_{k=1}^{N} a_{k} r_{n_{k}}^{*}(x) / A_{N}\right.\right. \\
& \left.\left.+\mu \sum_{k=1}^{N} a_{k}^{*} s_{n_{k}}^{*}(x) / A_{N}\right)\right) d x .
\end{aligned}
$$

As in Theorem II, we apply the formula

$$
e^{z}=(1+z) \exp \left(z^{2} / 2+Q(z)\right)
$$

in which $Q(z)=o\left(z^{2}\right)$. Letting $z_{N, k}=i 2 M a_{k}\left[\lambda r_{n_{k}}^{*}(x)+n s_{n_{k}}^{*}(x)\right] / A_{N}, \quad k=1$, $2, \cdots, N$, we have

$$
\begin{aligned}
& \Phi_{N}(\lambda, \mu: E)=m(E)^{-1} \int_{E} \prod_{k=1}^{N}\left(1+z_{N, k}\right) \\
& \quad \cdot \exp \left(-2 M^{2} \sum_{k=1}^{N} a_{k}^{2}\left[\lambda r_{n_{k}}^{*}(x)+\mu s_{n_{k}}^{*}(x)\right]^{2} / A_{N}^{2}+\sum_{k=1}^{N} Q\left(z_{N . k}\right)\right) d x .
\end{aligned}
$$

For $(\lambda, \mu)$ in a rectangle $\Omega \equiv[\alpha \leqq \lambda \leqq \beta, \gamma \leqq \mu \leqq \delta]$ it can be shown that $\exp \left(\sum_{k=1}^{N} Q\left(z_{N, k}\right)\right)=\exp (o(1))$ uniformly. It can also be shown as in Theorem II that the remaining factors in (4.4) are uniformly bounded for $(\lambda, \mu)$ in a rectangle $\Omega$. It follows that the uniform convergence of $\Phi_{N}(\lambda, \mu: E)$ on $\Omega$ to a limit $\Phi(\lambda, \mu: E)$ is equivalent to the uniform convergence on $\Omega$ of the functions

$$
\Phi_{N}^{*}(\lambda, \mu: E)=m(E)^{-1} \int_{E} \prod_{k=1}^{N}\left(1+z_{N, k}\right) \exp \left(-2 M^{2}\left[\lambda^{2} \sum_{k=1}^{N} a_{k}^{2} r_{n_{k}}^{* 2}(x) / A_{N}^{2}\right.\right.
$$

$$
\left.\left.+2 \lambda \mu \sum_{k=1}^{N} a_{k}^{2} r_{n_{k}}^{*}(x) s_{n_{k}}^{*}(x) / A_{N}^{2}+\mu^{2} \sum_{k=1}^{N} a_{k}^{2} s_{n_{k}}^{* 2}(x) / A_{N}^{2}\right]\right) d x
$$


A triple application of Theorem I and its corollary may be made and it is clear that a subsequence $\left\{\theta_{n_{k}}(x)\right\}$ (and so automatically subsequences $\left.\left\{r_{n_{k}}^{*}(x)\right\},\left\{s_{n_{k}}^{*}(x)\right\}\right)$ may be chosen so that for $\left\{\theta_{n_{k}}\right\}$ or any further subsequences $\left\{\theta_{n_{k_{j}}}(x)\right\} \equiv\left\{r_{n_{k_{j}}}^{*}(x)\right\}+i\left\{s_{n_{k_{j}}}^{*}(x)\right\}$, there exists real-valued functions, $\bar{R}(x), \bar{Q}(x), \bar{S}(x)$, such that for any sequence of real numbers $\left\{a_{\mathbf{k}}^{2}\right\}$ satisfying conditions (4.2)

$$
\begin{aligned}
& \sum_{k=1}^{N} a_{k}^{2} r_{n_{k}}^{* 2}(x) / A_{N}^{2} \rightarrow \bar{R}(x) \quad \text { in measure on }[a, b], \\
& \sum_{k=1}^{N} a_{k}^{2} r_{n_{k}}^{2}(x) s_{n_{k}}^{*}(x) / A_{N}^{2} \rightarrow \bar{Q}(x) \text { in measure on }[a, b], \\
& \sum_{k=1}^{N} a_{k}^{2} s_{n_{k}}^{* 2}(x) / A_{N}^{2} \rightarrow \bar{S}(x) \text { in measure on }[a, b] .
\end{aligned}
$$

The functions $\bar{R}(x), \bar{Q}(x), \bar{S}(x)$ are the same for all subsequences of $\left\{\theta_{n_{k}}\right\}$ and may be assumed to satisfy

$$
0 \leqq \bar{R}(x), \quad|\bar{Q}(x)|, \quad \bar{S}(x) \leqq 1 / 4, \quad a \leqq x \leqq b .
$$

Also since $\int_{a}^{b}\left(r_{n_{k}}^{* 2}(x)+s_{n_{k}}^{* 2}(x)\right) d x=1 /(2 M)^{2} \int_{a}^{b} \theta_{n_{k}}(x) \bar{\theta}_{n_{k}}(x) d x=1 / 4 M^{2}$ for $k=1$, $2, \cdots$, and since (from Theorem I) the functions $\bar{R}(x)$ and $\bar{S}(x)$ are weak limits of $\left\{r_{n_{k}}^{* 2}(x)\right\}$ and $\left\{s_{n_{k}}^{* 2}(x)\right\}$, it follows that

$$
\int_{a}^{b}(\bar{R}(x)+\bar{S}(x)) d x=\frac{1}{4 M^{2}} .
$$

Defining $F_{N}(y, z: E)$ and $\Phi_{N}(\lambda, \mu: E)$ with these subsequences: $\left\{r_{n_{k}}\right\},\left\{s_{n_{k}}\right\}$, and also defining with these (or any subsequence thereof),

$$
\begin{aligned}
\Phi_{N}^{* *}(\lambda, \mu: E)= & \frac{1}{m(E)} \int_{E} \prod_{k=1}^{N}\left(1+i(2 M) a_{k}\left(\lambda r_{n_{k}}^{*}(x)+\mu s_{n_{k}}^{*}(x)\right) / A_{N}\right) \\
& \cdot \exp \left(-2 M^{2}\left[\lambda^{2} \bar{R}(x)+2 \lambda \mu \bar{Q}(x)+\mu^{2} \bar{S}(x)\right]\right) d x,
\end{aligned}
$$

we see that the boundedness of the integrand in (4.4), (4.5), and (4.6) and the convergence in measure on $[a, b]$ of

$$
\begin{aligned}
\exp \left(-2 M^{2}\left[\lambda^{2} \sum_{k=1}^{N} a_{k}^{2} r_{n_{k}}^{* 2}(x) / A_{N}^{2}+2 \lambda \mu \sum_{k=1}^{N} a_{k}^{2} r_{n_{k}}^{*}(x) s_{n_{k}}^{*}(x) / A_{N}^{2}\right.\right. & . \\
& \left.\left.+\mu^{2} \sum_{k=1}^{N} a_{k}^{2} a_{k}^{* 2}(x) / A_{N}^{2}\right]\right)
\end{aligned}
$$

to $\exp \left(-2 M^{2}\left[\lambda^{2} \bar{R}(x)+2 \lambda \mu \bar{Q}(x)+\mu^{2} \bar{S}(x)\right]\right)$ uniformly for $(\lambda, \mu) \in \Omega$, as $N \rightarrow \infty$, implies that for any $E, m(E)>0$ and any $\left\{a_{k}\right\}$ satisfying (4.2),

$$
\left|\Phi_{N}(\lambda, \mu: E)-\Phi_{N}^{* *}(\lambda, \mu: E)\right| \rightarrow 0 \quad \text { as } N \rightarrow \infty,
$$


uniformly for $(\lambda, \mu)$ in $\Omega$. From this it follows that if we define

$$
\Phi(\lambda, \mu: E)=\frac{1}{m(E)} \int_{E} \exp \left(-1 / 2\left(\lambda^{2} R(x)+2 \lambda \mu Q(x)+\mu^{2} S(x)\right)\right) d x
$$

where $R(x)=4 M^{2} \bar{R}(x), Q(x)=4 M^{2} \bar{Q}(x), S(x)=4 M^{2} S(x)$, and if a further refinement of

$$
\left\{\theta_{n_{k}}(x)\right\} \equiv\left\{r_{n_{k}}(x)+i s_{n_{k}}(x)\right\}
$$

can be found for which, when $F_{N}(y, z: E)$ and $\Phi_{N}^{* *}(\lambda, \mu: E)$ are defined with the new subsequence, we have

$$
\Phi_{N}^{* *}(\lambda, \mu: E) \rightarrow \Phi(\lambda, \mu: E)
$$

uniformly for $(\lambda, \mu) \in \Omega$, independently of set $E$ and sequences $\left\{a_{k}\right\}$ satisfying (4.2), we shall have proved the generalized pseudo-Gaussian theorem. This further refinement is obtained by expanding in series and employing the Riemann-Lebesgue Theorem as in Theorem II. The details will not be given here.

TheOREM V. Let $\left\{\theta_{n}(x)\right\}$ be an orthonormal, complex-valued, uniformly bounded system of functions on a finite interval $[a, b]$. Then there exists a subsequence $\left\{\theta_{n_{k}}(x)\right\}$ and real-valued functions $R(x), Q(x), S(x)$ satisfying $0 \leqq R(x)$, $|Q(x)|, S(x) \leqq M^{2}, \int_{a}^{b}(R(x)+S(x)) d x=1$, such that for any real sequence $\left\{a_{k}\right\}$ satisfying

(a) $A_{N}=\left(a_{1}^{2}+a_{2}^{2}+\cdots+a_{N}^{2}\right)^{1 / 2} \rightarrow \infty$ as $N \rightarrow \infty$,

(b) $a_{N}=o\left(A_{N}\right)$

and any measurable set $E \subset[a, b], m(E)>0$, the distributions $\left\{F_{N}(y, z: E)\right\}$ defined by (4.1) tend to a limiting distribution $\{F(y, z: E)\}$ at each point of continuity of the latter, and the characteristic function $\Phi(\lambda, \mu: E)$ of this limiting distribution has the pseudo-Gaussian form (4.7). Moreover, any subsequence $\left\{\theta_{n_{k_{j}}}(x)\right\}$ of $\left\{\theta_{n_{k}}(x)\right\}$ also has this property.

REMARKs. (1) The nonsingular normal distribution for a pair of random variables (cf. Cramér [2, pp. 263, 265, 288]) is given by

$$
\begin{aligned}
G(t, s) & =\frac{1}{2 \pi \sigma_{1} \sigma_{2}\left(1-\rho^{2}\right)^{1 / 2}} \int_{-\infty}^{t} \int_{-\infty}^{s} \exp \left(-1 / 2\left(1-\rho^{2}\right)\left[\left(x-m_{1}\right)^{2} / \sigma_{1}^{2}\right.\right. \\
& \left.\left.-2 \rho\left(x-m_{1}\right)\left(y-m_{2}\right) / \sigma_{1} \sigma_{2}+\left(y-m_{2}\right)^{2} / \sigma_{2}^{2}\right]\right) / d x d y
\end{aligned}
$$

in which the center of gravity is $\left(m_{1} m_{2}\right), \sigma_{1}$ and $\sigma_{2}$ are the standard deviations of the random variables, and $\rho$ is the coefficient of correlation. In the case of the normal distribution, $\rho=0$ implies that the variables are independent. The characteristic function of this nonsingular normal distribution is

$$
\Phi(\lambda, \mu)=\exp \left(i\left(m_{1} \lambda+m_{2} \mu\right)-\left(\sigma_{1}^{2} \lambda^{2}+2 \sigma_{1} \sigma_{2} \lambda \mu+\sigma_{2}^{2}{ }^{2}\right) / 2\right) .
$$


The limiting distributions of Theorem $\mathrm{V}$ will still deserve the title "pseudoGaussian" inasmuch as when $R(x) \equiv c_{1}, Q(x) \equiv c_{2} \neq 0$, and $S(x) \equiv c_{3} ; c_{1}, c_{2}, c_{3}$ being constants, expression (4.7) will represent the characteristic function of a two-dimensional, nonsingular, normal distribution of dependent random variables with center of mass $(0,0)$. If $Q(x) \equiv 0$, the variables are asymptotically independent. An example in which this occurs is the case of two lacunary trigonometric series,

$$
\sum_{0}^{\infty}\left(a_{k} \cos n_{k} x+b_{k} \sin n_{k} x\right) \text { and } \sum_{0}^{\infty}\left(a_{k}^{\prime} \cos m_{k} x+b_{k}^{\prime} \sin m_{k} x\right),
$$

in which the combined sequence of integers $\left\{m_{k}, n_{k}\right\}$ is still lacunary. Zygmund and Salem [9] show that the distributions corresponding to (4.1), but with sums $\sum_{1}^{N}$ and $\sum_{1}^{M}$ ( $M$ and $N$ not necessarily equal), $\left\{F_{N M}(y, z: E)\right\}$, tend to the two-dimensional Gaussian distribution of independent variables having characteristic function

$$
\exp \left(-1 / 2\left(\lambda^{2}+\mu^{2}\right)\right)=\exp \left(-\lambda^{2} / 2\right) \exp \left(-\mu^{2} / 2\right)
$$

(2) As an example of the dependent variable case of Theorem $V$ we consider the complex-valued orthonormal system

$$
\rho_{n}(x)=\frac{\left(\phi_{n}(x)+i \phi_{n}(x)\right)}{2^{1 / 2}}, \quad n=1,2,3, \cdots,
$$

defined by means of the Rademacher functions $\left(^{(5)}\right.$ on $[0,1]$, with uniform bound $M=1$. Consider the distributions $F_{N}(y, z: E)$ for this system in the special case $E=[0,1]$.

By arguments preceding (4.7) we know that for any subsequence $\left\{\rho_{n_{k}}(x)\right\}$ and any $\left\{a_{k}\right\}$ satisfying (4.2), the characteristic functions $\Phi_{N}(\lambda, \mu:[0,1])$ defined with the corresponding altered real and imaginary subsequences $\left\{\phi_{n_{k}}^{*}(x)\right\} \equiv\left\{\phi_{n_{k}}(x) / 2\right\}$, as in (4.3), may be uniformly approximated over any rectangle by

$$
\begin{array}{r}
\Phi_{N}^{* *}(\lambda, \mu:[0,1])=\int_{0}^{1} \exp \left(-\left(\lambda^{2} \bar{R}(x)+2 \lambda \mu \bar{Q}(x)+\mu^{2} \bar{S}(x)\right)\right) \\
\cdot \prod_{k=1}^{N}\left(1+\frac{i 2 a_{k}\left(\lambda \phi_{n_{k}}^{*}(x)+\mu \phi_{n_{k}}^{*}(x)\right)}{2^{1 / 2} A_{N}}\right) d x
\end{array}
$$

where $\bar{R}(x), \bar{Q}(x), \bar{S}(x)$ are each the limit in measure of

$$
\sum_{k=1}^{N} a_{k}^{2} \phi_{n_{k}}^{* 2}(x) / A_{N}^{2}=\sum_{k=1}^{N} a_{k}^{2} \phi_{n_{k}}^{2}(x) / 4 A_{N}^{2} .
$$

But for any subsequence $\left\{\phi_{n_{k}}(x)\right\}$ of the Rademacher system and any $\left\{a_{k}^{2}\right\}$,

Ibid. Footnote 3. 


$$
\sum_{k=1}^{N} a_{k}^{2} \phi_{n_{k}}^{2}(x) / 4 A_{N}^{2}=\sum_{k=1}^{N} a_{k}^{2} / 4 A_{N}^{2}=\frac{1}{4}, \quad N=1,2,3, \cdots,
$$

and so $\bar{R}(x) \equiv \bar{Q}(x) \equiv S(x) \equiv 1 / 4$, and

$$
\begin{aligned}
& \Phi_{N}^{* *}(\lambda, \mu:[0,1]) \\
& \quad=\exp \left(-1 / 4\left(\lambda^{2}+2 \lambda \mu+\mu^{2}\right)\right) \int_{0}^{1} \prod_{k=1}^{N}\left(1+\frac{i a_{k}(\lambda+\mu) \phi_{n_{k}}^{*}(x)}{2^{1 / 2} A_{N}}\right) d x .
\end{aligned}
$$

If the product in the integrand is expanded by the rule (3.12), we obtain, after term-wise integration, a collection of terms which, with the exception of the initial term, 1 , are of the form

$$
\text { [coefficient in } \lambda \text { and } \mu]\left[\int_{0}^{1} \phi_{n_{k_{1}}}(x) \phi_{n_{k_{2}}}(x) \cdots \phi_{n_{k_{j}}}(x) d x\right]
$$

with all $n_{k_{i}}$ distinct. But such an integrand is a Walsh function $\psi_{r}(x), r>1$, and so has integral zero over $[0,1]$. This proves that uniformly over any rectangle $\Omega$,

$$
\Phi_{N}(\lambda, \mu:[0,1]) \rightarrow \exp \left(-1 / 4\left(\lambda^{2}+2 \lambda \mu+\mu^{2}\right)\right) \quad \text { as } N \rightarrow \infty .
$$

If now the Theorem $\mathrm{V}$ were applied to obtain a particular subsequence $\left\{\rho_{n_{k}}(x)\right\}$ which would give the result for any set $E$ of positive measure, the foregoing remark would imply that the corresponding distributions $F_{N}(y, z: E)$ converge for $E \equiv[0,1]$ to a nonsingular normal distribution whose characteristic function is $\exp \left(-1 / 4\left(\lambda^{2}+2 \lambda \mu+\mu^{2}\right)\right)$ of the dependent type.

(3) In the same manner the uniformly bounded orthonormal system, $\rho_{n}^{*}(x)=\left(\phi_{2 n-1}(x)+i \phi_{2 n}(x)\right) / 2^{1 / 2}, \quad n=1,2,3, \cdots$, contains a subsystem $\left\{\rho_{n_{k}}^{*}(x)\right\}$ which will determine distributions $F_{N}(y, z: E)$ such that for $E \equiv[0,1]$, the limiting distribution is of the independent normal type with characteristic function $\exp \left(-1 / 4\left(\lambda^{2}+\mu^{2}\right)\right)$. This follows from the details in Remark (2) and the additional fact that $Q(x) \equiv 0$ since $\left\{\phi_{2 n-1}(x) \cdot \phi_{2 n}(x)\right\}$ is a uniformly bounded system converging weakly (Riemann-Lebesgue theorem) to zero. By Theorem I there then exists a subsystem $\left\{\phi_{2 n_{k}-1}(x) \cdot \phi_{2 n_{k}}(x)\right\}$ such that $\sum_{k=1}^{N} a_{k}^{2} \phi_{2 n_{k-1}}(x) \cdot \phi_{2 n_{k}}(x) / A_{N}$ converges in measure to $Q(x) \equiv 0$. This is the way $Q(x)$ was obtained in Remark (2).

(4) A difference between the systems $\rho_{n}(x)$ and $\rho_{n}{ }^{*}(x)$ of the preceding remarks is that the Riemann-Lebesgue condition on the combined real and imaginary components, that is,

$$
\lim _{x \rightarrow \infty} \int_{a}^{b} f(x) r_{n_{k}}(x) s_{n_{k}}(x) d x=0 \quad \text { for all } f(x) \in L,
$$

is satisfied by the latter but not by the former. This condition is a sufficient 
condition for obtaining, in Theorem $\mathrm{V}, Q(x) \equiv 0$ in characteristic function (4.7). This may be called the asymptotically independent case.

For, if $\left\{\theta_{n_{k}}(x)\right\}$ is the chosen subsequence of $\left\{\theta_{n}(x)\right\}$ for which Theorem V is valid, and if $\left\{\theta_{n}(x)\right\}$ has real and imaginary sequences satisfying (4.8), then at the stage at which Theorem I applied to obtain $Q(x)$ with

$$
\sum_{k=1}^{N} a_{k}^{2} r_{n_{k}}^{*}(x) s_{n_{k}}^{*}(x) / A_{N}^{2} \rightarrow \bar{Q}(x) \text { in measure }
$$

we could declare $\bar{Q}(x) \equiv 0$ by the uniqueness of the weak limit in (4.8) and the role of the weak limit in the proof of Theorem I. Thus we would get (4.7) with $Q(x) \equiv 0$ as the limiting characteristic function in Theorem V.

The condition (4.8) then divides complex systems into two classes; the first class (i.e., sequences satisfying (4.8)) includes many common systems, e.g., $(\cos n x+i \sin n x) / 2 \pi, n=1,2,3, \cdots$, but excludes cases in which total dependence (identity) exists between the random variables, $\sum_{k=1}^{N} a_{k} r_{n_{k}}(x) / A_{N}$, $\sum_{k=1}^{N} a_{k} s_{n_{k}}(x) / A_{N}$, e.g. $(\cos n x+i \cos n x) /(2 \pi)^{1 / 2}$.

Finally, using Rademacher functions it is possible to establish the following result.

THEOREM VI. Let $R(x)$ and $S(x)$ be any two nonnegative, bounded, realvalued functions on $[0,1]$ such that $\int_{0}^{1}(R(x)+S(x)) d x=1$. Then there exists a complex-valued, 'uniformly bounded, orthonormal system $\left\{\theta_{n}(x)\right\}=\left\{r_{n}(x)\right.$ $\left.+i s_{n}(x)\right\}$ on $[0,1]$ such that for any sequence of real numbers $\left\{a_{k}\right\}$ satisfying

(a) $A_{N}=\left(a_{1}^{2}+\cdots+a_{N}^{2}\right)^{1 / 2} \rightarrow \infty$, as $N \rightarrow \infty$,

(b) $a_{N}=o\left(A_{N}\right)$,

and any measurable set $E \subset[0,1], m(E)>0$, the distribution functions formed with the real sequence $\left\{r_{n}(x)\right\}$ and imaginary sequence $\left\{s_{n}(x)\right\}$,

$$
F_{N}(y, z: E)=\frac{m\left(\left\{x \in E \mid \sum_{k=1}^{N} a_{k} r_{k}(x) / A_{N} \leqq y \text { and } \sum_{k=1}^{N} a_{k} s_{k}(x) / A_{N} \leqq z\right\}\right)}{m(E)},
$$

tend to a limiting distribution $F(y, z: E)$ whose characteristic function is

$$
\Phi(\lambda, \mu: E)=\frac{1}{m(E)} \int_{E} \exp \left(-1 / 2\left[\lambda^{2} R(x)+\mu^{2} S(x)\right]\right) d x .
$$

\section{BIBLIOGRAPHY}

1. S. Banach and S. Saks, Sur la convergence forte dans les champs $L^{p}$, Studia Math. vol. 2 (1930) pp. 51-57.

2. Harald Cramér, Mathematical methods of statistics, Princeton University Press, 1946.

3. N. J. Fine, On the Walsh functions, Trans. Amer. Math. Soc. vol. 65 (1949) pp. 372-414.

4. M. Kac, On the distribution of values of sums of the type $\sum f(2 t)$, Ann. of Math. (2) vol. 47 (1946) pp. 33-49. 
5. J. W. Lindeberg, Eine neue Herleitung des exponential Gesetzes in der Warscheinlichkeitsrechnung, Math. Zeit. vol. 15 (1922) p. 211.

6. M. Loève, Fundamental limit theorems of probability theory, Ann. Math. Statist. vol. 1 (1950) pp. 321-338.

7. R. E. A. C. Paley, A remarkable series of orthogonal functions, Proc. London Math. Soc. vol. 34 (1933) pp. 241-279.

8. F. Riesz, Über Systeme integrierbarer Funktionen, Math. Ann. vol. 69 (1910) pp. 449-497.

9. R. Salem and A. Zygmund, On lacunary trigonometric series, Proc. Nat. Acad. Sci. U.S.A. vol. 33 (1947) pp. 333-338.

10. A. Zygmund, Trigonometrical series, Warszawa-Lwow, Monografie Matematyczne, 1935.

University of Chicago,

Chicago, Ill. 